Portland State University

PDXScholar

Electrical and Computer Engineering Faculty

Publications and Presentations

$1-1-2009$

\title{
Radiative Transfer Theory Applied to Ocean Bottom Modeling
}

Lisa M. Zurk

Portland State University, zurkl@pdx.edu

Jorge Quijano

Portland State University

Follow this and additional works at: https://pdxscholar.library.pdx.edu/ece_fac

Part of the Electrical and Computer Engineering Commons

Let us know how access to this document benefits you.

\section{Citation Details}

Quijano, J. E., \& Zurk, L. M. (2009). Radiative transfer theory applied to ocean bottom modeling. The Journal of the Acoustical Society of America, 126, 1711.

This Article is brought to you for free and open access. It has been accepted for inclusion in Electrical and Computer Engineering Faculty Publications and Presentations by an authorized administrator of PDXScholar. Please contact us if we can make this document more accessible: pdxscholar@pdx.edu. 


\title{
Radiative transfer theory applied to ocean bottom modeling
}

\author{
Jorge E. Quijano ${ }^{\text {a) }}$ and Lisa M. Zurk \\ Department of Electrical Engineering, Northwest Electromagnetics and Acoustics Research Laboratory, \\ Portland State University, Portland, Oregon 97201-0751
}

(Received 27 October 2008; revised 30 April 2009; accepted 20 July 2009)

\begin{abstract}
Research on the propagation of acoustic waves in the ocean bottom sediment is of interest for active sonar applications such as target detection and remote sensing. The interaction of acoustic energy with the sea floor sublayers is usually modeled with techniques based on the full solution of the wave equation, which sometimes leads to mathematically intractable problems. An alternative way to model wave propagation in layered media containing random scatterers is the radiative transfer (RT) formulation, which is a well established technique in the electromagnetics community and is based on the principle of conservation of energy. In this paper, the RT equation is used to model the backscattering of acoustic energy from a layered elastic bottom sediment containing distributions of independent scatterers due to a constant single frequency excitation in the water column. It is shown that the RT formulation provides insight into the physical phenomena of scattering and conversion of energy between waves of different polarizations.
\end{abstract}

(C) 2009 Acoustical Society of America. [DOI: 10.1121/1.3203992]

PACS number(s): 43.30.Ft, 43.20.Bi, 43.20.Fn, 43.30.Vh [JJM] Pages: 1711-1723

\section{INTRODUCTION}

Research on the interaction of acoustic energy with the sea floor has gained importance due to its role on the performance of sonar systems in shallow water and the demand for more accurate models for remote sensing of the sea floor. In the first case, a better understanding of this interaction can help to differentiate sources in the water column from bottom reverberation and improve estimates of bottom reflection loss for navy applications. In the latter case, it can enhance current techniques for inversion of geoacoustic parameters and/or provide physical understanding of the structure of the scattered field for bottom probing applications. The goal of this work is to introduce the radiative transfer (RT) formulation as an alternative method to the classic wave theory approach for computation of volume scattering.

Ocean bottom sediments are usually modeled as discrete layers with rough interfaces containing volume inhomogeneities. Acoustic backscattering is divided in rough surface scattering due to the contrast at the water-sediment interface and volume scattering due to subbottom inhomogeneities. ${ }^{1}$ Although there are models that unify volume and rough surface scattering into a single formulation, ${ }^{2,3}$ a simple model that handles multiple layered sediments with embedded random scatterers remains a challenge.

Several formulations based on the classic wave theory have been developed to predict sediment backscattering, and most of them are based on the integral method. ${ }^{1,3,4}$ This technique consists of writing the sound speed and the density as constant mean values with random perturbations that depend on the position in the media. The resulting homogeneous wave equation for the perturbed media can be recast into a heterogeneous wave equation with a "source" term that is a

\footnotetext{
${ }^{\text {a) }}$ Author to whom correspondence should be addressed. Electronic mail: jorgeq@pdx.edu
}

function of the perturbations. With the integral method, the statement of the problem is exact, ${ }^{3}$ but the solution of the integral equation requires approximations that might limit its range of applicability.

Examples of such approximations are the assumption of weak scatterers ${ }^{2,5}$ or small fractional volumes. ${ }^{6}$ Due to high sediment attenuation these assumptions simplify the mathematical formulation by considering only single scattering events. In most cases these models have shown good agreement with experimental backscattering data, but they have underestimated volume scattering in certain environments ${ }^{7}$ and it has been suggested that this might be due to neglecting of multiple scattering.

Other common approximation in current volume scattering models is the assumption of shallow acoustic penetration, which allows to ignore contributions from deeper scattering features but it constrains the model to frequencies higher than $10 \mathrm{kHz}$ and shallow grazing angles. ${ }^{1}$ Also, most of the classic models neglect the often complicated contribution of shear waves, which is not a good approximation for certain types of consolidated elastic media. ${ }^{8}$

Contrary to the classic wave theory, the RT formulation works with the propagation of energy rather than the fields that carry it, and it is suggested that in general, its solution is much simpler than the integral method approach from classic wave theory. ${ }^{9}$ RT theory has been a dynamic area of research in electromagnetic remote sensing, optic sensing, and more recently it has been adapted to acoustic waves to model the propagation of longitudinal and shear waves in elastic media, with applications in seismics ${ }^{10}$ and ultrasound. ${ }^{11}$ In those applications, the environment consists of parallel layers with embedded random scatterers, and this similarity with the seabed motivates this work. ${ }^{12}$

Despite the common presentation of RT theory as a phenomenological or heuristic statement of energy conservation, the RT theory for parallel-plane homogeneous layers with 
random discrete scatterers can also be derived from formal principles of wave theory. ${ }^{13,14}$ More recent developments include several approximate techniques to handle densely packed discrete scatterers, ${ }^{15-17}$ gradients of the refractive index of background media, ${ }^{18}$ horizontally variable layered environments, ${ }^{19,20}$ and time-dependent broadband excitations. $^{21,22}$

The RT equation presented in this work is the full elastic solution which includes the effect of multiple scattering, attenuation due to lossy scatterers or lossy background media, and transformation of waves of different polarizations and multiple parallel layers. Wave theory is still utilized to estimate the scattering characteristics of the random particles, but once the scattering and absorption cross sections are defined, the problem reduces to solving the RT equation. Then, the scattering media can be characterized by an extinction factor (that accounts for scattering and loss mechanisms) and an emission factor (which describes the scattering of energy from neighboring particles into the direction of observation).

In this paper, the main equations for the RT formulation for acoustics as derived by Turner and Weaver ${ }^{11}$ are summarized and applied to layered media containing low density distributions of spherical voids. The focus of this paper is on the steady-state RT formulation, which corresponds to a monochromatic constant excitation. The notation introduced in this paper will be extended in the future to the solution of the transient RT formulation ${ }^{22}$ required to simulate finite length pulses with the spectral characteristics commonly used in sea experiments. It is shown that the RT solution provides straightforward intuition into the physical problem and that the effect of each scattering process can be isolated.

This paper is organized as follows: Sec. II explains the scalar RT equation from a phenomenological perspective. In Sec. III, the vector RT equation utilized in acoustics is introduced for a finite layer with reflective boundaries, and Sec. IV summarizes the procedure to solve this equation. Section $\mathrm{V}$ presents the equations for the plane wave reflection/ transmission coefficients that are utilized in this paper as boundary conditions for the water-elastic and the elasticelastic interface. In Sec. VI, the relation between the specific intensity and the power flux is explained, and in Sec. VII simulations for different combinations of background attenuation and layering structure are presented. Section VIII corresponds to conclusions and a summary of the capabilities and limitations of the RT model for random media.

\section{THE CONCEPT OF RT}

The RT theory was initially formulated by astrophysicists for study of stellar spectra, ${ }^{23}$ and several methods for the solution of the RT equation have been proposed. Some solution methods take advantage of symmetry of the random media, and in this section the RT equation is summarized for the specific case of parallel planes with discrete scatterers, as described in the literature. ${ }^{11,24}$ With parallel-plane media, the intensity $I(\theta, \phi, z)$ propagating in the random media is a function of depth $z$, azimuth $\phi$, and elevation angle $\theta$.

The interaction of energy with the layered media is illustrated in Fig. 1, where the total intensity can be divided

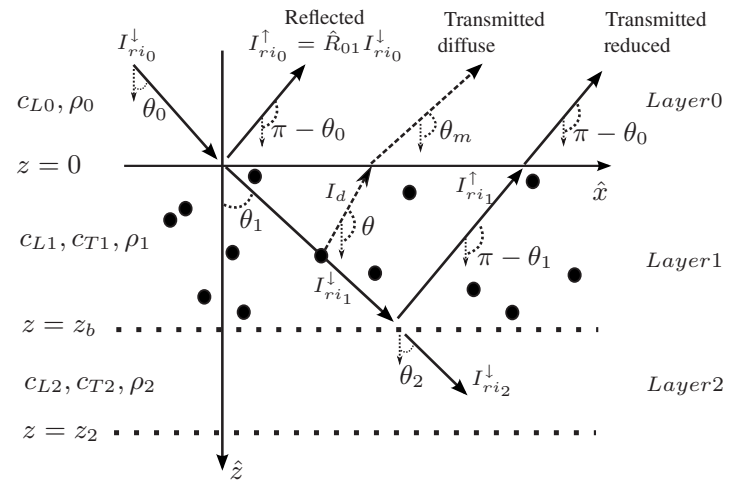

FIG. 1. Diagram of the reduced intensity $I_{\text {ri }}$ (solid arrows) and the diffuse intensity $I_{d}$ (dashed arrows) in a layer containing random scatterers. In this paper, $\theta_{a}$ is the diffracted angle for a wave in media $a$ measured with respect to the $\hat{z}$ axis, with $\theta_{0}$ denoting the incident angle of the energy from the source in layer 0 . The reflection coefficient for specific intensity, $\hat{R}_{a b}$, corresponds to a wave traveling and bouncing back to media $a$ when the boundary $a \mid b$ is reached. Layer 2 is an infinite half space with $z_{2}=\infty$.

into two components: the reduced intensity $I_{\mathrm{ri}}$, which represents the coherent energy that travels with a constant direction, and the diffuse intensity $I_{d}$, which propagates in all directions.

The reduced intensity is attenuated due to its interaction with the scatterers and the multiple reflections in layer 1 as well as absorption within the media. From conservation laws, part of the attenuated energy will escape layer 1 and this is determined by the transmission coefficients $\hat{T}_{10}$ and $\hat{T}_{12}$ from media 1 to media 0 and 2, respectively (the subindex notation follows Tsang et al. ${ }^{25}$ and the symbol ${ }^{\wedge}$ refers to the transmission/reflection coefficients for specific intensities, as explained in Sec. V). Since the rest of the attenuated energy becomes the diffuse intensity, the reduced intensity can be regarded as a source of diffuse intensity, and the amount of diffuse intensity depends on the nature of the volume scatterers.

In this paper, flat interfaces are assumed and the Fresnel reflection coefficients are utilized. For a single interaction with the bottom, the total upward intensity $I(\theta, \phi, z)$ in the direction $\left(\theta_{m}>\pi / 2, \phi_{m}\right)$ in layer 0 at $z=0^{-}$is given by

$$
\begin{aligned}
I\left(\theta_{m}>\right. & \left.\frac{\pi}{2}, \phi_{m}, 0^{-}\right) \\
= & \hat{T}_{10} I_{d}\left(\theta>\frac{\pi}{2}, \phi_{m}, 0^{+}\right)+\left[\hat{R}_{01} I_{\text {rio }}^{\downarrow}\left(\theta_{0}, \phi_{0}, 0^{-}\right)\right. \\
& \left.+\hat{T}_{10} I_{\text {ri }}^{\uparrow}\left(\pi-\theta_{1}, \phi_{0}, 0^{+}\right)\right] \delta\left(\theta_{m}-\left(\pi-\theta_{0}\right)\right) \delta\left(\phi_{m}-\phi_{0}\right),
\end{aligned}
$$

where $(\theta, \phi)$ are the elevation and azimuth angles of the diffuse intensity, $I_{d}$ and $I_{\text {ri1 }}^{\uparrow}$ are the diffuse and upward reduced intensities in layer 1, respectively, $I_{\text {rio }}^{\downarrow}$ is the incident intensity, and $\delta$ is the Kronecker operator to indicate that the coherent intensity can be observed only in the specular direction $\left(\theta_{0}, \phi_{0}\right)$. The reduced intensity includes the reflection coefficient between layers 1 and $2, \hat{R}_{12}$, and the attenuation of energy due to interaction with the random scatterers. Mathematically, it can be written as 


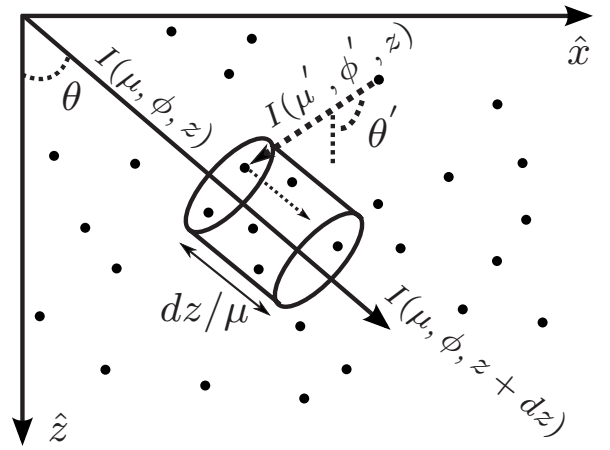

FIG. 2. Diagram of the interaction of an energy beam traveling in the $(\theta, \phi)$ direction with scatterers within a differential volume. The intensity at the output of the volume, $I(\mu, \phi, z+d z)$, results from the attenuation of the input intensity $I(\mu, \phi, z)$ and the reinforcement due to energy coming from other directions $\left(\theta^{\prime}, \phi^{\prime}\right)$.

$$
I_{\mathrm{ri}_{1}}^{\uparrow}\left(\pi-\theta_{1}, \phi_{0}, 0\right)=\hat{T}_{01} \hat{R}_{12} I_{\mathrm{ri}_{0}}^{\downarrow}\left(\theta_{0}, \phi_{0}, 0\right) \exp \left[-2 \frac{\eta \sigma z_{b}}{\cos \theta_{1}}\right],
$$

where $z_{b}$ is the thickness of the layer with random scatterers, $\eta$ is the density of scatterers, and $\sigma$ is known as the extinction coefficient, which is a characteristic of the scatterers and the background attenuation and will be defined later. The angle $\theta_{1}$ results from diffraction when energy in layer 0 with an incidence angle $\theta_{0}$ is transmitted to layer 1 , according to Snell's law. As expected, the intensity related to volume scattering $\left(I_{d}\right)$ will be zero if $\theta_{0}$ is greater than the critical angle at the fluid-water interface since no energy can penetrate into layer 1 .

In the RT literature it is a common practice to define the specific intensity as $I(\mu, \phi, z)$, where $\mu=\cos \theta$ is negative for upward going intensity and positive for downward going intensity. This convention will be adopted in this paper.

Figure 2 shows a differential volume containing a distribution of $\eta$ scatterers per $\mathrm{m}^{3}$.

The change in the total intensity $I(\mu, \phi, z)$ as a function of the traveled distance $d z / \mu$ and the energy that arrives from other directions $\left(\theta^{\prime}, \phi^{\prime}\right)$ can be expressed as ${ }^{11,24}$

$$
\begin{aligned}
\frac{\partial I(\mu, \phi, z)}{\partial(z / \mu)}= & -[\eta(\kappa+\nu)+2 \alpha] I(\mu, \phi, z) \\
& +\frac{\eta}{4 \pi} \int_{-1}^{1} \int_{0}^{2 \pi} p\left(\mu, \phi ; \mu^{\prime}, \phi^{\prime}\right) I\left(\mu^{\prime}, \phi^{\prime}, z\right) d \mu^{\prime} d \phi^{\prime},
\end{aligned}
$$

where $I(\mu, \phi, z)=I_{\mathrm{ri}}(\mu, \phi, z)+I_{d}(\mu, \phi, z)$ includes the reduced and diffuse intensities, $\kappa$ and $\nu$ are the scattering and absorption cross sections of a single particle normalized by $4 \pi$, respectively, $\alpha$ is the attenuation of the background media in $\mathrm{Np} / \mathrm{m}$, and $p\left(\mu, \phi ; \mu^{\prime}, \phi^{\prime}\right)$ is the scattering strength into direction $(\theta, \phi)$ for a single particle when the incident energy arrives from direction $\left(\theta^{\prime}, \phi^{\prime}\right)$. Following the definition by Turner and Weaver, ${ }^{11} \kappa$ $=(1 / 4 \pi) \int_{\Omega=4 \pi} p\left(\mu, \phi ; \mu^{\prime}, \phi^{\prime}\right) d \Omega$ where $\int_{\Omega=4 \pi}\langle\cdot\rangle d \Omega$ indicates an integral over a solid angle of $4 \pi$.

The form of Eq. (3) assumes independent scattering, where the total scattering is the product $\eta(\kappa+\nu)$. If the frac- tional volume is large enough (typically $5 \%-10 \%$ or greater), the dense media radiative transfer formulation is used instead, where the total scattering is substituted by an effective scattering parameter. $^{15,16}$

From Eq. (3), the intensity within the volume is decreased by $[\eta(\kappa+\nu)+2 \alpha]$ and increased by the contribution of energy coming from all directions and coupling into the $(\theta, \phi)$ direction. This is represented by the integral over all possible $\theta^{\prime}$ and $\phi^{\prime}$ angles.

The extinction cross section $\sigma=(\kappa+\nu)+2 \alpha / \eta$ is a measurement of the amount of coherent energy that is transformed into diffuse energy due to scattering and absorption of the background media and the particles.

\section{RT EQUATION IN ELASTIC MEDIA}

In this section, the procedure developed by Turner and Weaver ${ }^{11}$ to obtain a RT for ultrasound is outlined and modified for the case of a finite layer with reflecting boundaries at the top and bottom.

For acoustics, the specific intensity $I(\theta, \phi, z)$ can be divided in longitudinal and shear waves. From the definition of Stokes parameters, five specific intensities can be defined: $I_{L}$, $I_{x}$, and $I_{y}$ represent the total energy due to longitudinal, shear horizontal, and shear vertical waves, respectively, and $I_{U}$ and $I_{V}$ represent correlations between the shear waves. As in Sec. II the total intensity is the summation of the reduced and the diffuse intensities. Since these intensities have different polarizations, they are orthogonal to each other and Eq. (3) can be applied to each one independently:

$$
\begin{aligned}
\mu \frac{\partial I_{L}(\mu, \phi, z)}{\partial z}= & -\eta \sigma_{L} I_{L}(\mu, \phi, z) \\
& +\frac{\eta}{4 \pi} \int_{-1}^{1} \int_{0}^{2 \pi}\left[\sum_{b} P_{L b}\left(\mu, \phi ; \mu^{\prime}, \phi^{\prime}\right)\right. \\
& \left.\times I_{b}\left(\mu^{\prime}, \phi^{\prime}, z\right)\right] d \mu^{\prime} d \phi^{\prime}
\end{aligned}
$$

for the longitudinal component and

$$
\begin{aligned}
\mu \frac{\partial I_{a}(\mu, \phi, z)}{\partial z}= & -\eta \sigma_{T} I_{a}(\mu, \phi, z) \\
& +\frac{\eta}{4 \pi} \int_{-1}^{1} \int_{0}^{2 \pi}\left[\sum_{b} P_{a b}\left(\mu, \phi ; \mu^{\prime}, \phi^{\prime}\right)\right. \\
& \left.\times I_{b}\left(\mu^{\prime}, \phi^{\prime}, z\right)\right] d \mu^{\prime} d \phi^{\prime}
\end{aligned}
$$

for any of the four transversal components, where $b$ $\in[L, x, y, U, V]$ and $a$ is any of the shear specific intensities $(x, y, U$, or $V)$. The longitudinal and shear extinction cross sections are defined as $\sigma_{L}=\kappa_{L}+v_{L}+2 \alpha_{L} / \eta$ and $\sigma_{T}=\kappa_{T}+v_{T}$ $+2 \alpha_{T} / \eta$, respectively. The term $P_{a b}\left(\mu, \phi ; \mu^{\prime}, \phi^{\prime}\right)$ represents the coupling of energy from a wave with $b$ polarization into a wave with $a$ polarization. Conceptually, it is similar to the scattering function $p\left(\mu, \phi ; \mu^{\prime}, \phi^{\prime}\right)$ for the scalar case, except that $P_{a b}\left(\mu, \phi ; \mu^{\prime}, \phi^{\prime}\right)$ includes the rotation of Stokes parameters about an axis, which is a step required to align the 
incident and scattered intensities with an arbitrary scattering plane. $^{11,13}$

Equations (4) and (5) have the same form, and it is convenient to define a vector RT equation that can be solved more efficiently. Also, in RT literature the variable $\tau=\eta \kappa_{T} z$ is defined as a normalized depth. After this substitution,

$$
\begin{aligned}
\mu \frac{\partial \underline{I}(\mu, \phi, \tau)}{\partial \tau}= & -\underline{\tilde{\sigma}} \underline{I}(\mu, \phi, \tau) \\
& +\frac{1}{4 \pi \kappa_{T}} \int_{-1}^{1} \int_{0}^{2 \pi} \underline{\mathrm{P}}\left(\mu, \phi ; \mu^{\prime}, \phi^{\prime}\right) \\
& \times \underline{I}\left(\mu^{\prime}, \phi^{\prime}, \tau\right) d \mu^{\prime} d \phi^{\prime},
\end{aligned}
$$

where the underbar denotes vector/matrix quantities and

$$
\begin{aligned}
& \underline{I}(\mu, \phi, \tau)=\left(\begin{array}{c}
I_{L} \\
I_{y} \\
I_{x} \\
U \\
V
\end{array}\right), \\
& \widetilde{\sigma}=\left(\begin{array}{ccccc}
\widetilde{\sigma}_{L} & 0 & 0 & 0 & 0 \\
0 & \widetilde{\sigma}_{T} & 0 & 0 & 0 \\
0 & 0 & \widetilde{\sigma}_{T} & 0 & 0 \\
0 & 0 & 0 & \tilde{\sigma}_{T} & 0 \\
0 & 0 & 0 & 0 & \widetilde{\sigma}_{T}
\end{array}\right),
\end{aligned}
$$

with $\widetilde{\sigma}_{L}=\sigma_{L} / \kappa_{L}$ and $\tilde{\sigma}_{T}=\sigma_{T} / \kappa_{L}$. The term $\underline{P}\left(\mu, \phi ; \mu^{\prime}, \phi^{\prime}\right)$ is the $5 \times 5$ Mueller matrix ${ }^{11}$ for a single scatterer:

$$
\underline{\mathrm{P}}\left(\mu, \phi ; \mu^{\prime}, \phi^{\prime}\right)=\left(\begin{array}{ccccc}
P_{L L} & P_{L y} & P_{L x} & P_{L U} & P_{L V} \\
P_{y L} & P_{y y} & P_{y x} & P_{y U} & P_{y V} \\
P_{x L} & P_{x y} & P_{x x} & P_{x U} & P_{x V} \\
P_{U L} & P_{U y} & P_{U x} & P_{U U} & P_{U V} \\
P_{V L} & P_{V y} & P_{V x} & P_{V U} & P_{V V}
\end{array}\right)
$$

[the angular dependence of the elements in the matrix $\underline{P}\left(\mu, \phi ; \mu^{\prime}, \phi^{\prime}\right)$ has been suppressed for brevity]. Except for the addition of the background attenuation $\alpha$ and the assumption of steady-state conditions, Eq. (6) is identical to Eq. (48) from Turner and Weaver. ${ }^{11}$ In analogy to the scalar case,

$$
\begin{aligned}
& \kappa_{L}=\frac{1}{4 \pi} \int_{\Omega=4 x}\left[P_{L L}+P_{x L}+P_{y L}\right] d \Omega, \\
& \kappa_{T}=\frac{1}{8 \pi} \int_{\Omega=8 x}\left[P_{L y}+P_{y y}+P_{x y}+P_{L x}+P_{y x}+P_{x x}\right] d \Omega .
\end{aligned}
$$

For a finite layer 1 of thickness $z_{b}$ and reflecting boundaries, the boundary conditions are defined as

$\underline{I}\left(\mu>0, \phi, 0^{+}\right)=\underline{I}_{s r c}+\underline{I}_{10}\left(\mu>0, \phi, 0^{+}\right), \quad z=0^{+}$downward,

$\underline{I}\left(\mu<0, \phi, \tau_{b}^{-}\right)=\underline{I}_{12}\left(\mu<0, \phi, \tau_{b}^{-}\right), \quad z=z_{b}^{-}$upward,

where

$$
\underline{I}_{s r c}\left(\mu, \phi, 0^{+}\right)=\left[\begin{array}{c}
\hat{T}_{01}^{L L} K_{L 0} \delta\left(\theta-\theta_{1}^{L_{1}}\right) \\
\hat{T}_{01}^{L y} K_{L 0} \delta\left(\theta-\theta_{1}^{y_{1}}\right) \\
0 \\
0 \\
0
\end{array}\right]
$$

results from the coupling of energy from the water column into the sediment. $K_{L 0}$ is the amplitude of the incident power flux at $z=0$ due to an acoustic source in the water column. This flux is assumed to be a collimated beam in the direction $\left(\theta_{o}^{L_{1}}, \phi_{o}\right)$ and it diffracts into longitudinal and shear specific intensities in the sediment, with amplitude determined by the specific intensity transmission coefficients $\hat{T}_{01}^{L L}$ (longitudinalto-longitudinal) and $\hat{T}_{01}^{L y}$ (longitudinal-to-shear vertical); there is no longitudinal-to-shear horizontal coupling, so $\hat{T}_{01}^{L x}$ $=0$. As a convention, the superindex in the reflection/ transmission coefficients indicates the change in polarization and the subindex indicates the layers at the interface.

The angle of the diffracted waves is indicated by $\mu_{1}^{L_{1}}$ $=\cos \theta_{1}^{L_{1}}$ and $\mu_{1}^{y_{1}}=\cos \theta_{1}^{y_{1}}$, where as in Fig. 1 , the subindex corresponds to the layer. The superindex has been introduced to indicate whether the angle describes a longitudinal or a shear vertical intensity. As explained in Sec. IV, multiple interactions of the intensity with the boundaries of the layer result in the alignment of the coherent intensity along several directions of propagation that are indicated with a numerical value in the superindex. For example, $\theta_{1}^{L_{1}}$ and $\theta_{1}^{L_{2}}$ correspond both to longitudinal coherent intensities in layer 1, traveling in two different angles that are labeled as $L_{1}$ and $L_{2}$. Similarly, $\theta_{1}^{y_{1}}$ and $\theta_{1}^{y_{2}}$ are shear vertical coherent intensities in layer 1 along two angles labeled as $y_{1}$ and $y_{2}$.

The vectors $I_{10}$ and $I_{12}$ are the reflected specific intensities at $\tau=0$ and $\tau=\tau_{b}$, respectively, and are defined as

$$
\begin{aligned}
\underline{I}_{10}\left(\mu>0, \phi, 0^{+}\right) & =\left[\begin{array}{c}
\hat{R}_{10}^{L L} I_{L}\left(-\mu, \phi, 0^{+}\right)+\hat{R}_{10}^{y L} I_{y}\left(-\mu_{1}^{y_{A}}, \phi, 0^{+}\right) \\
\hat{R}_{10}^{L y} I_{L}\left(-\mu_{1}^{L_{A}}, \phi, 0^{+}\right)+\hat{R}_{10}^{y y} I_{y}\left(-\mu, \phi, 0^{+}\right) \\
I_{x}\left(-\mu, \phi, 0^{+}\right) \\
\hat{R}_{10}^{U U} I_{U}\left(-\mu, \phi, 0^{+}\right)+\hat{R}_{10}^{V U} I_{V}\left(-\mu, \phi, 0^{+}\right) \\
\hat{R}_{10}^{U V} I_{U}\left(-\mu, \phi, 0^{+}\right)+\hat{R}_{10}^{V V} I_{V}\left(-\mu, \phi, 0^{+}\right)
\end{array}\right],
\end{aligned}
$$

$$
\begin{array}{r}
\underline{I}_{12}\left(\mu<0, \phi, \tau_{b}^{-}\right) \\
=\left[\begin{array}{c}
\hat{R}_{12}^{L L} I_{L}\left(-\mu, \phi, \tau_{b}^{-}\right)+\hat{R}_{12}^{y L} I_{y}\left(\mu_{1}^{y_{A}}, \phi, \tau_{b}^{-}\right) \\
\hat{R}_{12}^{L y} I_{L}\left(\mu_{1}^{L_{A}}, \phi, \tau_{b}^{-}\right)+\hat{R}_{12}^{y y} I_{y}\left(-\mu, \phi, \tau_{b}^{-}\right) \\
I_{x}\left(-\mu, \phi, \tau_{b}^{-}\right) \\
\hat{R}_{12}^{U U} I_{U}\left(-\mu, \phi, \tau_{b}^{-}\right)+\hat{R}_{12}^{V U} I_{V}\left(-\mu, \phi, \tau_{b}^{-}\right) \\
\hat{R}_{12}^{U V} I_{U}\left(-\mu, \phi, \tau_{b}^{-}\right)+\hat{R}_{12}^{V V} I_{V}\left(-\mu, \phi, \tau_{b}^{-}\right)
\end{array}\right],
\end{array}
$$

where $\hat{R}_{c d}^{a b}$ is the reflection coefficient for an incident wave with polarization $a$ into a wave with polarization $b$ at the boundary between media $c$ and $d$. The variables $\mu_{1}^{L_{A}}$ $=\cos \theta_{1}^{L_{A}}$ and $\mu_{1}^{y_{A}}=\cos \theta_{1}^{y_{A}}$ indicate off-axis contribution due 

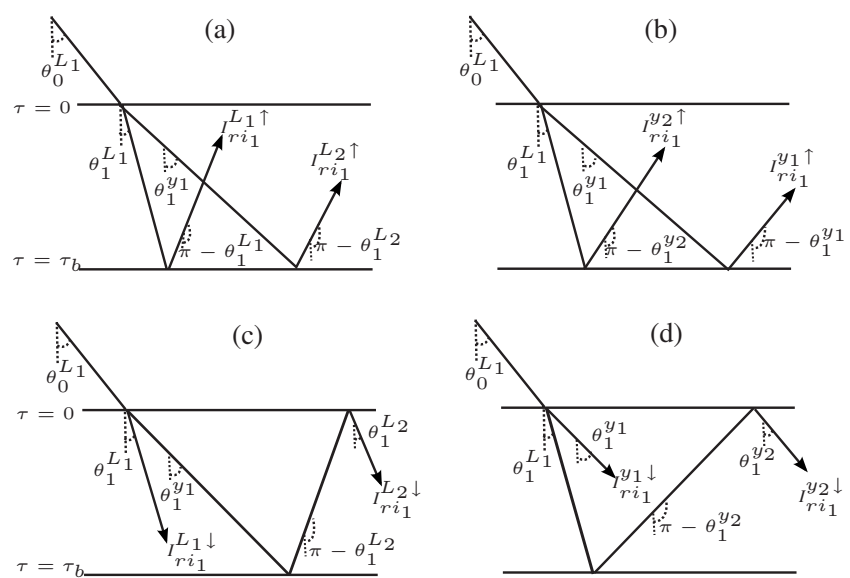

FIG. 3. The reduced intensity can be collimated in eight different directions (a) The upward longitudinal intensities $I_{\mathrm{ri}_{1}}^{L_{1} \uparrow}$ and $I_{\mathrm{ri}_{1}}^{L_{2} \uparrow}$ result from the transformations $L-L-L$ and $L-y-L$, respectively, where $L$ stands for longitudinal and $y$ for shear vertical, (b) Similar to (a) for $I_{\mathrm{ri}_{1}}^{y_{1} \uparrow}(L-y-y)$ and $I_{\mathrm{ri}_{1}}^{y_{1} \uparrow}$ $(L-L-y)$; (c) The downward longitudinal intensities $I_{\mathrm{ri}_{1}}^{L_{1} \downarrow}$ and $I_{\mathrm{ri}_{1}}^{L_{2} \downarrow}$ correspond to the transformations $L-L$ and $L-y-L-L$, respectively, (d) Similar to (c) for $I_{\mathrm{ri}_{1}}^{y_{1} \downarrow}(L-y)$ and $I_{\mathrm{ri}_{1}}^{y_{2} \downarrow}(L-L-y-y)$.

to conversion from longitudinal and shear vertical polarizations and are defined as

$$
\begin{aligned}
& \theta_{1}^{L_{A}}=\sin ^{-1}\left(\frac{c_{L 1}}{c_{T 1}} \sin \theta\right), \quad \theta_{1}^{y_{A}}=\sin ^{-1}\left(\frac{c_{T 1}}{c_{L 1}} \sin \theta\right), \\
& \theta=\cos ^{-1}|\mu| .
\end{aligned}
$$

\section{SOLUTION TO THE RT EQUATION}

In general, Eq. (6) must be solved numerically but in some cases (like single scattering solutions ${ }^{11}$ ), analytical solutions can also be obtained. For any of those approaches, the reduced intensity must be found first so it can be used as a source for the diffuse intensity.

\section{A. The reduced intensity for a finite layer}

For the general case of a finite layer with reflecting boundaries, the reduced intensity has upward and downward components. ${ }^{24}$ Due to the refraction of longitudinal waves into shear waves and vice versa, the resulting reduced intensity can be collimated along eight different angles, as shown in Fig. 3.

Each diagram in Fig. 3 represents a possible combination of multiple reflections of the energy within the sediment. The notation for the reduced intensity is similar to the notation for angles in Eq. (12): the subindex $\mathrm{ri}_{1}$ stands for reduced intensity in layer 1 , and the superindices $L_{1} \downarrow$ and $L_{2} \downarrow$ stand for downward longitudinal intensities collimated with two different angles ( $L_{1}$ and $L_{2}$, respectively), and a similar interpretation applies to the other terms. A closed form expression for each combination of multiple reflections can be found by writing an infinite summation of terms and simplifying the resulting geometric series, ${ }^{24}$ which is a useful result for the steady-state solution that is the subject of this paper. However, writing each of the interactions as a separate term will allow the extension of the notation in preparation for the transitory solution of the RT equation, which can be accom- plished by following the procedure found in the literature ${ }^{22}$ and it will be the subject of a future communication.

The first eight interactions of the coherent excitation with the layer boundaries are

$$
\begin{aligned}
& I_{\mathrm{ri}_{1}}^{L_{1} \uparrow}(\mu, \phi, \tau)=A_{1}^{L_{1} \uparrow}(\mu) e^{-\widetilde{\sigma}_{L} \tau / \mu} \delta\left(\theta-\left(\pi-\theta_{1}^{L_{1}}\right)\right) \delta\left(\phi-\phi_{o}\right), \\
& I_{\mathrm{ri}_{1}}^{L_{2} \uparrow}(\mu, \phi, \tau)=A_{1}^{L_{2} \uparrow}(\mu) e^{-\widetilde{\sigma}_{L} \tau / \mu} \delta\left(\theta-\left(\pi-\theta_{1}^{L_{2}}\right)\right) \delta\left(\phi-\phi_{o}\right), \\
& I_{\mathrm{ri}_{1}}^{y_{1} \uparrow}(\mu, \phi, \tau)=A_{1}^{y_{1} \uparrow}(\mu) e^{-\widetilde{\sigma}_{T} \tau / \mu} \delta\left(\theta-\left(\pi-\theta_{1}^{y_{1}}\right)\right) \delta\left(\phi-\phi_{o}\right), \\
& I_{\mathrm{ri}_{1}}^{y_{1} \uparrow}(\mu, \phi, \tau)=A_{1}^{y_{1} \uparrow}(\mu) e^{-\widetilde{\sigma}_{T} \tau / \mu} \delta\left(\theta-\left(\pi-\theta_{1}^{y_{2}}\right)\right) \delta\left(\phi-\phi_{o}\right),
\end{aligned}
$$

where

$$
\begin{aligned}
& A_{1}^{L_{1} \uparrow}(\mu)=K_{L 0} \hat{T}_{01}^{L L} \hat{R}_{12}^{L L} \exp \left[\frac{2 \widetilde{\sigma}_{L} \tau_{b}}{\mu}\right], \\
& A_{1}^{L_{2} \uparrow}(\mu)=K_{L 0} \hat{T}_{01}^{L y} \hat{R}_{12}^{y L} \exp \left[-\tau_{b}\left(\frac{\widetilde{\sigma}_{T}}{\mu_{1}^{y_{1}}}-\frac{\widetilde{\sigma}_{L}}{\mu}\right)\right], \\
& A_{1}^{y_{1} \uparrow}(\mu)=K_{L 0} \hat{T}_{01}^{L y} \hat{R}_{12}^{y y} \exp \left[\frac{2 \widetilde{\sigma}_{T} \tau_{b}}{\mu}\right], \\
& A_{1}^{y_{2} \uparrow}(\mu)=K_{L 0} \hat{T}_{01}^{L L} \hat{R}_{12}^{L y} \exp \left[-\tau_{b}\left(\frac{\widetilde{\sigma}_{L}}{\mu_{1}^{L_{1}}}-\frac{\widetilde{\sigma}_{T}}{\mu}\right)\right]
\end{aligned}
$$

are the amplitude terms. Similarly, the expressions for the downward reduced intensities are

$$
\begin{aligned}
& I_{\mathrm{ri}_{1}}^{L_{1} \downarrow}(\tau, \mu, \phi)=A_{1}^{L_{1} \downarrow}(\mu) e^{-\widetilde{\sigma}_{L} / \mu} \delta\left(\theta-\theta_{1}^{L_{1}}\right) \delta\left(\phi-\phi_{o}\right), \\
& I_{\mathrm{ri}_{1}}^{L_{2} \downarrow}(\tau, \mu, \phi)=A_{1}^{L_{2} \downarrow}(\mu) e^{-\widetilde{\sigma}_{L} \tau / \mu} \delta\left(\theta-\theta_{1}^{L_{2}}\right) \delta\left(\phi-\phi_{o}\right), \\
& I_{\mathrm{ri}_{1}}^{y_{1} \downarrow}(\tau, \mu, \phi)=A_{1}^{y_{1} \downarrow}(\mu) e^{-\widetilde{\sigma}_{T} \tau / \mu} \delta\left(\theta-\theta_{1}^{y_{1}}\right) \delta\left(\phi-\phi_{o}\right), \\
& I_{\mathrm{ri}_{1}}^{y_{2} \downarrow}(\tau, \mu, \phi)=A_{1}^{y_{2} \downarrow}(\mu) e^{-\widetilde{\sigma}_{T} \tau / \mu} \delta\left(\theta-\theta_{1}^{y_{2}}\right) \delta\left(\phi-\phi_{o}\right),
\end{aligned}
$$

where

$$
\begin{aligned}
& A_{1}^{L_{1} \downarrow}(\mu)=K_{L 0} \hat{T}_{01}^{L L}, \\
& A_{1}^{L_{2} \downarrow}(\mu)=K_{L 0} \hat{T}_{01}^{L y} \hat{R}_{12}^{y L} \hat{R}_{11}^{L L} \exp \left[-\tau_{b}\left(\frac{\widetilde{\sigma}_{T}}{\mu_{1}^{y_{1}}}+\frac{\widetilde{\sigma}_{L}}{\mu}\right)\right], \\
& A_{1}^{y_{1} \downarrow}(\mu)=K_{L 0} \hat{T}_{01}^{L y}, \\
& A_{1}^{y_{2} \downarrow}(\mu)=K_{L 0} \hat{T}_{01}^{L L} \hat{R}_{12}^{L y} \hat{R}_{11}^{y y} \exp \left[-\tau_{b}\left(\frac{\widetilde{\sigma}_{T}}{\mu_{1}^{L_{1}}}+\frac{\widetilde{\sigma}_{L}}{\mu}\right)\right] .
\end{aligned}
$$

The variables $\mu_{1}^{L_{1}}, \mu_{1}^{y_{1}}, \mu_{1}^{L_{2}}, \mu_{1}^{y_{2}}$ are the cosines of the angles

$$
\theta_{1}^{L_{1}}=\sin ^{-1}\left[\frac{c_{L 1}}{c_{L 0}} \sin \theta_{0}^{L_{1}}\right], \quad \theta_{1}^{y_{1}}=\sin ^{-1}\left[\frac{c_{T 1}}{c_{L 0}} \sin \theta_{0}^{L_{1}}\right],
$$




$$
\theta_{1}^{L_{2}}=\sin ^{-1}\left[\frac{c_{L 1}}{c_{T 1}} \sin \theta_{1}^{y_{1}}\right], \quad \theta_{1}^{y_{2}}=\sin ^{-1}\left[\frac{c_{T 1}}{c_{L 1}} \sin \theta_{1}^{L_{1}}\right],
$$

where $c_{L a}$ and $c_{T a}$ are the longitudinal and shear sound speeds in the ath layer, respectively.

\section{B. The RT equation for the diffuse specific intensity}

The procedure to solve Eq. (6) consists of writing the total specific intensity as the summation of the reduced and the diffuse intensity:

$$
\underline{I}(\mu, \phi, \tau)=\underline{I}_{\mathrm{ri}}(\mu, \phi, \tau)+\underline{I}_{d}(\mu, \phi, \tau),
$$

where

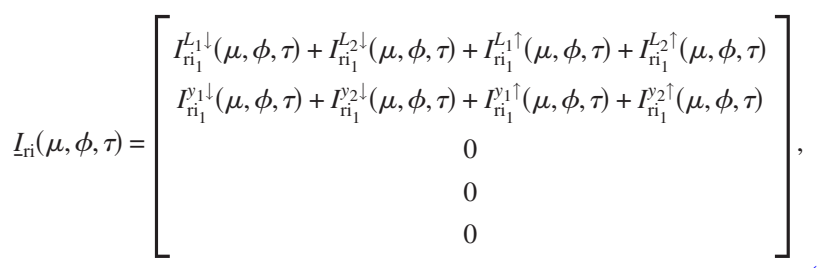

and

$$
\underline{I}_{d}(\mu, \phi, \tau)=\left[\begin{array}{c}
I_{d_{1}}^{L}(\mu, \phi, \tau) \\
I_{d_{1}}^{y}(\mu, \phi, \tau) \\
I_{d_{1}}^{x}(\mu, \phi, \tau) \\
I_{d_{1}}^{U}(\mu, \phi, \tau) \\
I_{d_{1}}^{V}(\mu, \phi, \tau)
\end{array}\right]
$$

is the vector of diffuse intensities to be found. Note that for any specific angle $\mu$ only one term in the sum (22) will be non-zero due to the $\delta$ operands in Eqs. (16) and (18). Substitution of Eq. (21) into Eq. (6) results in an expression for $\underline{I}_{d}(\mu, \phi, \tau)$ with sources of longitudinal and transversal specific intensities:

$$
\begin{aligned}
\mu \frac{\partial \underline{I}_{d}(\mu, \phi, \tau)}{\partial \tau} & \\
= & -\widetilde{\sigma} \underline{I}_{d}(\mu, \phi, \tau) \\
& +\frac{1}{4 \pi \kappa_{T}}\left[\int_{-1}^{1} \int_{0}^{2 \pi} \underline{\mathrm{P}}\left(\mu, \phi ; \mu^{\prime}, \phi^{\prime}\right) \underline{I}_{d}\left(\mu^{\prime}, \phi^{\prime}, \tau\right) d \mu^{\prime} d \phi^{\prime}\right] \\
& +\underline{S}_{1}^{L_{1} \downarrow}(\mu, \phi) e^{-\widetilde{\sigma}_{L} \tau / \mu_{1}^{L_{1}}}+\underline{S}_{1}^{L_{2} \downarrow}(\mu, \phi) e^{-\widetilde{\sigma}_{L} \tau / \mu_{1}^{L_{2}}} \\
& +\underline{S}_{1}^{y_{1} \downarrow}(\mu, \phi) e^{-\widetilde{\sigma}_{T} \tau / \mu_{1}^{y_{1}}}+\underline{S}_{1}^{y_{2} \downarrow}(\mu, \phi) e^{-\widetilde{\sigma}_{T} \tau / \mu_{1}^{y_{2}}} \\
& +\underline{\mathrm{S}}_{1}^{L_{1} \uparrow}(\mu, \phi) e^{\widetilde{\sigma}_{L} \tau / \mu_{1}^{L_{1}}}+\underline{S}_{1}^{L_{2} \uparrow}(\mu, \phi) e^{\widetilde{\sigma}_{L} \tau / \mu_{1}^{L_{2}}} \\
& +\underline{S}_{1}^{y_{1} \uparrow}(\mu, \phi) e^{\widetilde{\sigma}_{T} \tau / \mu_{1}^{y_{1}}}+\underline{S}_{1}^{y_{2} \uparrow}(\mu, \phi) e^{\widetilde{\sigma}_{T} \tau / \mu_{1}^{y_{2}}},
\end{aligned}
$$

where

$$
\begin{aligned}
& \underline{\mathrm{S}}_{1}^{L 1 \downarrow}(\mu, \phi)=\frac{1}{4 \pi \kappa_{T}} \underline{\mathrm{P}}\left(\mu, \phi ; \mu_{1}^{L_{1}}, \phi_{o}\right)\left(\begin{array}{c}
A_{1}^{L 1 \downarrow}\left(\mu_{1}^{L_{1}}\right) \\
0 \\
0 \\
0 \\
0
\end{array}\right) \text {, } \\
& \underline{S}_{1}^{L_{2} \downarrow}(\mu, \phi)=\frac{1}{4 \pi \kappa_{T}} \underline{P}\left(\mu, \phi ; \mu_{1}^{L_{2}}, \phi_{o}\right)\left(\begin{array}{c}
A_{1}^{L_{2} \downarrow}\left(\mu_{1}^{L_{2}}\right) \\
0 \\
0 \\
0 \\
0
\end{array}\right) \text {, } \\
& \underline{\mathrm{S}}_{1}^{y_{1} \downarrow}(\mu, \phi)=\frac{1}{4 \pi \kappa_{T}} \underline{\mathrm{P}}\left(\mu, \phi ; \mu_{1}^{y_{1}}, \phi_{o}\right)\left(\begin{array}{c}
0 \\
A_{1}^{y_{1} \downarrow}\left(\mu_{1}^{y_{1}}\right) \\
0 \\
0 \\
0
\end{array}\right) \text {, } \\
& \underline{\mathrm{S}}_{1}^{y_{2} \downarrow}(\mu, \phi)=\frac{1}{4 \pi \kappa_{T}} \underline{\mathrm{P}}\left(\mu, \phi ; \mu_{1}^{y_{2}}, \phi_{o}\right)\left(\begin{array}{c}
0 \\
A_{1}^{y_{2} \downarrow}\left(\mu_{1}^{y_{2}}\right) \\
0 \\
0 \\
0
\end{array}\right) \text {, }
\end{aligned}
$$

and the upward source terms $\underline{S}_{1}^{L_{1} \uparrow}(\mu, \phi), \quad \underline{S}_{1}^{L_{2}}(\mu, \phi)$, $\underline{S}_{1}^{y_{1} \uparrow}(\mu, \phi)$, and $\underline{S}_{1}^{L y_{2} \uparrow}(\mu, \phi)$ can be obtained from Eqs. (25)-(28) by substituting " $\downarrow$ " by " $\uparrow$ " and $\mu_{1}^{a_{b}}$ by $-\mu_{1}^{a_{b}}$ in the phase matrix $\underline{\mathrm{P}}\left(\mu, \phi ; \mu_{1}^{a_{b}}, \phi_{o}\right)$.

For the diffuse intensity in Eq. (24) the same boundary conditions as in Eqs. (13) and (14) can be used, and the solution of the diffuse intensity is outlined in the Appendix.

\section{PLANE WAVE REFLECTION COEFFICIENTS FOR AN ELASTIC-ELASTIC INTERFACE}

For simplicity, in this paper the plane wave reflection and transmission coefficients for elastic media are utilized to define the boundary conditions for the RT differential equation. For the water-sediment interface, the incident longitudinal wave from the water column results in a reflected longitudinal wave and transmitted longitudinal and shear vertical waves. Expressions for the reflection and transmission coefficients at the water-sediment interface were presented elsewhere. ${ }^{12}$ For the sand-limestone interface, shear and longitudinal waves are supported in both interfaces. From Brekhovskikh, ${ }^{26}$ the reflection/transmission coefficients for a longitudinal wave in media 1 with incidence angle $\theta_{1}^{L}$ are

$$
\begin{aligned}
\Delta R_{12}^{L L}= & \Upsilon_{1}^{2}-\beta_{1} \Upsilon_{2}^{2} / \alpha_{1}+\left(\alpha_{2} / \beta_{2}\right)\left(B_{1}^{2}-\beta_{1} B_{2}^{2} / \alpha_{1}\right) \\
& +m\left(\beta_{2} / \beta_{1}-\alpha_{2} / \alpha_{1}\right)\left(\kappa_{1}^{4} / 4 \zeta^{4}\right), \\
-\Delta / 2 R_{12}^{L y} & =\Upsilon_{1} \Upsilon_{2}+\left(\alpha_{2} / \beta_{2}\right) B_{1} B_{2}, \\
\Delta T_{12}^{L L}= & \left(\kappa_{2}^{2} / \zeta^{2}\right)\left(\Upsilon_{1}-B_{2}\right),
\end{aligned}
$$




$$
\Delta T_{12}^{L y}=\left(\kappa_{2}^{2} / \zeta^{2}\right)\left(\Upsilon_{2}+\alpha_{2} B_{1} / \beta_{2}\right)
$$

where

$$
\begin{aligned}
\Delta= & \Upsilon_{1}^{2}+\beta_{1} \Upsilon_{2}^{2} / \alpha_{1}+\left(\alpha_{2} / \beta_{2}\right)\left(B_{1}^{2}+\beta_{1} B_{2}^{2} / \alpha_{1}\right) \\
& +m\left(\beta_{2} / \beta_{1}+\alpha_{2} / \alpha_{1}\right)\left(\kappa_{1}^{4} / 4 \zeta^{4}\right), \\
\Upsilon_{1}= & n^{2}-m p_{2} / \zeta, \\
\Upsilon_{2}= & \left(n^{2} p_{1}-m p_{2}\right) / \beta_{1}, \\
B_{1}= & \left(n^{2}-m\right) \beta_{2} / \zeta, \\
B_{2}= & \left(\beta_{2} / \beta_{1}\right)\left(n^{2} p_{1} / \zeta-m\right),
\end{aligned}
$$

and

$$
\begin{aligned}
& n=\frac{c_{T 1}}{c_{T 2}}, \quad m=\frac{\rho_{1}}{\rho_{2}}, \quad \zeta=\left(\frac{\omega}{c_{L 1}}\right) \sin \theta_{1}^{L}, \\
& \beta_{1}=\left(\frac{\omega}{c_{T 1}}\right) \cos \theta_{1}^{y}, \quad \beta_{2}=\left(\frac{\omega}{c_{T 2}}\right) \cos \theta_{2}^{y}, \\
& \alpha_{1}=\left(\frac{\omega}{c_{L 1}}\right) \cos \theta_{1}^{L}, \quad \alpha_{2}=\left(\frac{\omega}{c_{L 2}}\right) \cos \theta_{2}^{L}, \\
& p_{1}=\left[\zeta^{2}-\left(\frac{\omega}{c_{T 1}}\right)^{2} \frac{1}{2}\right] \zeta^{-1}, \quad p_{2}=\left[\zeta^{2}-\left(\frac{\omega}{c_{T 2}}\right)^{2} \frac{1}{2}\right] \zeta^{-1} .
\end{aligned}
$$

The angles $\theta_{1}^{L}, \theta_{1}^{y}, \theta_{2}^{L}$, and $\theta_{2}^{y}$ are related by Snell's law:

$$
\frac{\sin \theta_{1}^{L}}{c_{L 1}}=\frac{\sin \theta_{1}^{y}}{c_{T 1}}=\frac{\sin \theta_{2}^{L}}{c_{L 2}}=\frac{\sin \theta_{2}^{y}}{c_{T 2}} \text {. }
$$

A vertical shear wave will also excite longitudinal and vertical shear waves in both elastic media, and it will be characterized by the corresponding $R_{12}^{y L}, R_{12}^{y y}, T_{12}^{y L}$, and $T_{12}^{y y}$ coefficients. ${ }^{26}$ Finally, a shear horizontal wave in the sediment can only excite reflected and transmitted shear horizontal waves, so it is completely characterized by two coefficients $R_{12}^{x x}$ and $T_{12}^{x x}$.

Since the main quantity for the RT equation is the specific intensity, the coefficients for the specific intensity must be utilized instead. Table I shows the relationship between the plane wave coefficients and its counterpart for specific intensity in an elastic-elastic interface. For the watersediment interface, the corresponding coefficients can be found in the literature. ${ }^{27}$

Table II shows the value of the geoacoustic parameters for a sandy sediment and a limestone layer.

\begin{tabular}{|c|c|c|}
\hline Inc. & Power coefficient & Specific intensity \\
\hline \multirow[t]{3}{*}{$\mathrm{L}$} & $\begin{array}{c}\tilde{R}_{12}^{L L}=\left|R_{12}^{L L}\right|^{2} \\
\tilde{R}_{12}^{L y}=\left|R_{12}^{L y}\right|^{2} \frac{\tan \theta_{1}^{L}}{\tan \theta_{1}^{y}}\end{array}$ & $\begin{array}{c}\hat{R}_{12}^{L L}=\tilde{R}_{12}^{L L} \\
\hat{R}_{12}^{L y}=\widetilde{R}_{12}^{L y} \frac{c_{L 1}^{2}}{c_{T 1}^{2}}\end{array}$ \\
\hline & $\tilde{T}_{12}^{L L}=\left|T_{12}^{L L}\right|^{2} \frac{\rho_{2} \tan \theta_{1}^{L}}{\rho_{1} \tan \theta_{2}^{L}}$ & $\hat{T}_{12}^{L L}=\tilde{T}_{12}^{L L} \frac{c_{L 1}^{2}}{c_{L 2}^{2}}$ \\
\hline & $\widetilde{T}_{12}^{L y}=\left|T_{12}^{L y}\right|^{2} \frac{\rho_{2} \tan \theta_{1}^{L}}{\rho_{1} \tan \theta_{2}^{y}}$ & $\hat{T}_{12}^{L y}=\widetilde{T}_{12}^{L y} \frac{c_{L 1}^{2}}{c_{T 2}^{2}}$ \\
\hline \multirow[t]{3}{*}{ SV } & $\begin{array}{c}\widetilde{R}_{12}^{y y}=\left|R_{12}^{y y}\right|^{2} \\
\widetilde{R}_{12}^{y L}=\left|R_{12}^{y L}\right|^{2} \frac{\tan \theta_{1}^{y}}{\tan \theta_{1}^{L}}\end{array}$ & $\begin{array}{c}\hat{R}_{12}^{y y}=\tilde{R}_{12}^{y y} \\
\hat{R}_{12}^{y L}=\widetilde{R}_{12}^{y L} \frac{c_{T 1}^{2}}{c_{L 1}^{2}}\end{array}$ \\
\hline & $\tilde{T}_{12}^{y y}=\left|T_{12}^{y y}\right|^{2} \frac{\rho_{2} \tan \theta_{1}^{y}}{\rho_{1} \tan \theta_{2}^{y}}$ & $\hat{T}_{12}^{y y}=\widetilde{T}_{12}^{y y} \frac{c_{T 1}^{2}}{c_{T 2}^{2}}$ \\
\hline & $\widetilde{T}_{12}^{y L}=\left|T_{12}^{y L}\right|^{2} \frac{\rho_{2} \tan \theta_{1}^{y}}{\rho_{1} \tan \theta_{2}^{L}}$ & $\hat{T}_{12}^{y L}=\tilde{T}_{12}^{y L} \frac{c_{T 1}^{2}}{c_{L 2}^{2}}$ \\
\hline $\mathrm{SH}$ & $\begin{array}{c}\widetilde{R}_{12}^{x x}=\left|R_{12}^{x x}\right|^{2} \\
\widetilde{T}_{12}^{x x}=\left|T_{12}^{x x}\right|^{2} \frac{\rho_{2} \tan \theta_{1}^{x}}{\rho_{1} \tan \theta_{2}^{x}}\end{array}$ & $\begin{array}{c}\hat{R}_{12}^{x x}=\hat{R}_{12}^{x x} \\
\hat{T}_{12}^{x x}=\widetilde{T}_{12}^{x x} \frac{c_{T 1}^{2}}{c_{T 2}^{2}}\end{array}$ \\
\hline
\end{tabular}

Simulations for the power transmission and reflection coefficients from a sand-limestone interface are shown in Figs. 4(a) and 4(b). The relations $\left|\widetilde{R}_{12}^{L L}\right|+\left|\widetilde{R}_{12}^{L y}\right|+\left|\widetilde{T}_{12}^{L L}\right|+\left|\widetilde{T}_{12}^{L y}\right|$ $=1$ and $\left|\widetilde{R}_{12}^{y L}\right|+\left|\widetilde{R}_{12}^{y y}\right|+\left|\widetilde{T}_{12}^{y L}\right|+\left|\widetilde{T}_{12}^{y y}\right|=1$ can be verified at any angle, but this relation does not hold for the specific intensity coefficients. Similar simulations for the water-sediment interface were presented in a previous paper. ${ }^{12}$

As discussed in Sec. IV the transmitted/reflected reduced intensity (shown in terms of transmission/reflection
TABLE I. Power ( ${ }^{\sim}$ ) and specific intensity $\left({ }^{\wedge}\right)$ reflection/transmission coefficients for the elastic-elastic interface.

coefficients in Fig. 4) gives rise to additional sources of coherent upward and downward intensities that have the potential to be transformed into diffuse intensity. The complex behavior of the transmission and reflection of energy at the sand-limestone interface modulates the amplitude of those sources. This will be evident in Sec. VII when the volume scattering from a finite layer on a water-sand-limestone arrangement is compared to the scattering levels with the limestone layer replaced by sand.

\section{CONSERVATION OF POWER FOR AN INCIDENT COHERENT BEAM}

One of the main features of the RT model is the conservation of power. This can be used to test the accuracy of the RT computer simulation and to provide the contribution of longitudinal and shear waves in units of power rather than specific intensity. The relationship between specific intensity $I_{f_{g}}^{e^{\uparrow}}(\mu, \phi, z)$ and the upward power flux $F_{f_{g}}^{e \uparrow}(z)$ normal to layer $g$ at any depth $z$ is ${ }^{13}$

TABLE II. Acoustic properties of the sediment, limestone, and water column used in this paper.

\begin{tabular}{cccc}
\hline \hline Variable & Sediment $^{\mathrm{a}}$ & Water & Limestone $^{\mathrm{b}}$ \\
\hline$\rho_{s}\left(\mathrm{~kg} / \mathrm{m}^{3}\right)$ & 2023.2 & 1027 & 2200 \\
$c_{L}(\mathrm{~m} / \mathrm{s})$ & 1689 & 1500 & 4390 \\
$c_{T}(\mathrm{~m} / \mathrm{s})$ & 117 & 0 & 2570 \\
$\alpha_{L}(\mathrm{~dB} / \mathrm{m}$ at $10 \mathrm{kHz})$ & 5.24 & 0 & 0.2 \\
$\alpha_{T}(\mathrm{~dB} / \mathrm{m}$ at $10 \mathrm{kHz})$ & 178 & 0 & 0.7 \\
\hline \hline
\end{tabular}

${ }^{a}$ References 28 and 29.

${ }^{\mathrm{b}}$ References 30 and 31 . 

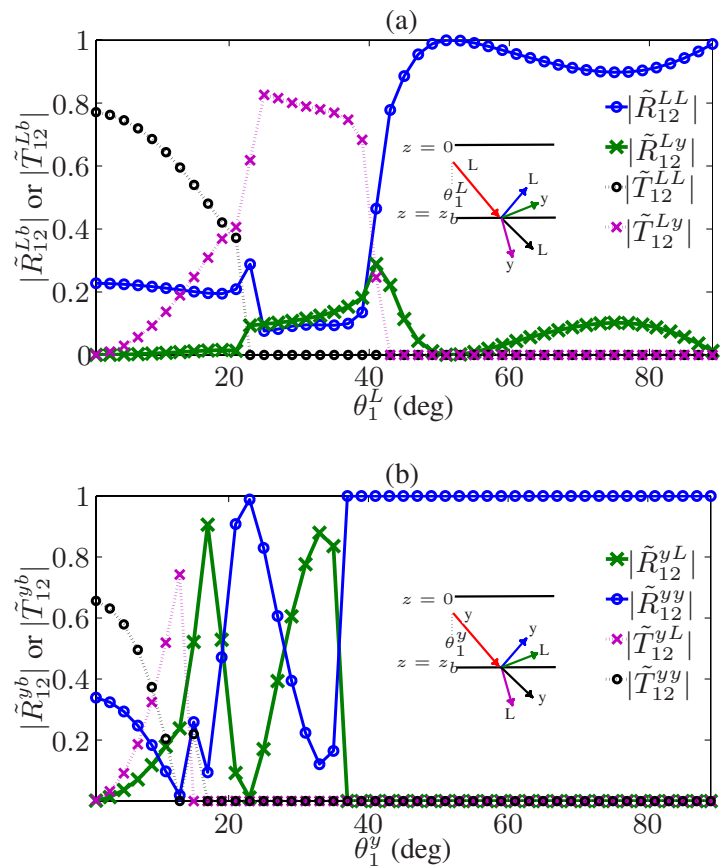

FIG. 4. (Color online) Power reflection/transmission coefficients at the sand-limestone interface. (a) Coefficients $\widetilde{R}_{12}^{L L}, \widetilde{R}_{12}^{L_{y}}, \widetilde{T}_{12}^{L L}$, and $\widetilde{T}_{12}^{L_{y}}$ for an incident longitudinal wave, as shown in the inserted diagram. (b) Coefficients $\widetilde{R}_{12}^{y y}, \widetilde{R}_{12}^{y L}, \hat{T}_{12}^{y y}$, and $\widetilde{T}_{12}^{y}$ for an incident shear vertical wave, as shown in the inserted diagram.

$$
F_{f_{g}}^{e \uparrow}(z)=\int_{2 \pi} I_{f_{g}}^{e \uparrow}(\mu, \phi, z) \mu d \Omega, \quad-1<\mu<0,
$$

where $d \Omega$ is a solid angle and $0<\phi<2 \pi$, the subindex $f$ refers to reduced (ri) or diffuse $(d)$ and the superindex $e$ could be $L_{1}, L_{2}, y_{1}$, etc. Similarly, the downward power flux normal to layer $g$ at any depth $z$ is

$$
F_{f_{g}}^{e \downarrow}(z)=\int_{2 \pi} I_{f_{g}}^{e \downarrow}(\mu, \phi, z) \mu d \Omega, \quad 0<\mu<1 .
$$

As an example,

$$
F_{\mathrm{ri}_{1}}^{L_{1} \uparrow}(z)=\int_{2 \pi} I_{\mathrm{ri}_{1}}^{L_{\uparrow} \uparrow}(\mu, \phi, z) \mu d \Omega, \quad-1<\mu<0
$$

is the upward power flux due to the coherent longitudinal specific intensity $I_{\mathrm{ri}_{1}}^{L_{1} \uparrow}(\mu, \phi, z)$, while

$$
F_{d_{1}}^{L \downarrow}(z)=\int_{2 \pi} I_{d_{1}}^{L \downarrow}(\mu, \phi, z) \mu d \Omega, \quad 0<\mu<1
$$

is the downward flux due to the diffuse specific intensity $I_{d_{1}}^{L \downarrow}(\mu, \phi, z)$. If the attenuation of the background media in layer 1 is zero and the scatterers are lossless, the conservation of the normal component of power flux can be stated as

$$
\begin{array}{rlrl} 
& \left.\left(F_{\mathrm{ri}_{0}}^{L \downarrow}-F_{\mathrm{ri}_{0}}^{L \uparrow}\right)\right|_{z=0} & & \text { (incident-reflected) } \\
= & \left.\left(F_{d_{1}}^{L \uparrow}+F_{d_{1}}^{T \uparrow}\right)\right|_{z=0}+\left.\left(F_{d_{1} \downarrow}^{L \downarrow}+F_{d_{1} \downarrow}^{T \downarrow}\right)\right|_{z=z_{b}} & & \text { (diffuse/volume scat.) } \\
+ & \left.\left(F_{\mathrm{ri}_{1}}^{L 1 \uparrow}+F_{\mathrm{ri}_{1}}^{L 2 \uparrow}\right)\right|_{z=0}+\left.\left(F_{\mathrm{ri}_{1}}^{L 1 \downarrow}+F_{\mathrm{ri}_{1}}^{L 2 \downarrow}\right)\right|_{z=z_{b}} & & \text { (coherent } L) \\
+ & \left.\left(F_{\mathrm{ri}_{1}}^{y \uparrow}+F_{\mathrm{ri}_{1}}^{y 2 \uparrow}\right)\right|_{z=0}+\left.\left(F_{\mathrm{ri}_{1}}^{y 1}+F_{\mathrm{ri}_{1}}^{y 2 \downarrow}\right)\right|_{z=z_{b}} & \text { (coherent } T)
\end{array}
$$

where

$$
\begin{aligned}
& F_{\mathrm{ri}_{0}}^{L_{1} \downarrow}(z)=\int_{2 \pi} I_{\mathrm{ri}_{0}}^{L_{1} \downarrow}(\mu, \phi, z) \cos \theta d \Omega, \\
& F_{r}^{L_{\uparrow} \uparrow}(z)=\int_{2 \pi} I_{\mathrm{ri}_{0}}^{L_{1} \uparrow}(\mu, \phi, z) \cos \theta d \Omega
\end{aligned}
$$

are the normal fluxes due to the coherent incident and reflected specific intensities in the water column. The relation in Eq. (41) states that all energy provided to the sediment must manifest itself in either diffuse or upward/downward reduced intensities. The incident and reflected specific intensities are defined as

$$
\begin{aligned}
& I_{\mathrm{ri}_{0}}^{L_{1} \downarrow}(\mu, \phi, \tau)=K_{L 0} \delta\left(\theta-\theta_{0}^{L_{1}}\right) \delta\left(\phi-\phi_{0}\right), \\
& I_{\mathrm{ri}_{0}}^{L_{1} \uparrow}(\mu, \phi, \tau)=\hat{R}_{01}^{L L} K_{L 0} \delta\left(\theta-\left(\pi-\theta_{0}^{L_{1}}\right)\right) \delta\left(\phi-\phi_{0}\right) .
\end{aligned}
$$

In Sec. VII, simulated results are presented in terms of the normal power fluxes in Eq. (41).

\section{RESULTS}

Simulations were run for $K_{L 0}=1 \mathrm{~W} / \mathrm{m}^{2}$ with different combinations of sediment attenuation and boundary conditions. The scatterers are spherical cavities, and the scattering terms in Eq. (9) were computed using the Mie analytical solution [see Eq. (86) from Turner and Weaver ${ }^{27}$ ]. In all simulations, the frequency $f=10 \mathrm{kHz}$, the particle radius $a$ $=0.01 \mathrm{~m}, z_{b}=1 \mathrm{~m}$, and the particle density $\eta=2388$ scatterers $/ \mathrm{m}^{3}$.

Figures 5 and 6 were computed to show the conservation of the outward power flux that is normal to the boundaries of the layer containing scatterers when $\sigma_{L}=\kappa_{L}$ and $\sigma_{T}=\kappa_{T}$ (no background attenuation or scatterer absorption). In both cases, the conservation of the normal power flux established in Eq. (41) can be confirmed at each incidence angle $\theta_{0}^{L_{1}}$.

Comparison between Figs. 5 and 6 also shows the effect of the contrast between layer 1 and the bottom halfspace. Figure 5 corresponds to a sand bottom half space (no contrast between layers 1 and 2), while Fig. 6 shows the resulting power flux when the limestone bottom half space is introduced.

In Fig. 5, the selection of the same background material for layers 1 and 2 results in the suppression of the upward coherent specific intensities since $R_{12}^{L L}=R_{12}^{L y}=0$, and for this simulation, the amount of volume scattering coming out of the layer at $z=z_{b},\left.\quad\left(F_{d_{1}}^{L \downarrow}+F_{d_{b}}^{T \downarrow}\right)\right|_{z=z_{b}}$, is higher than the volume scattering at $z=0,\left.\quad\left(F_{d_{1}}^{L \uparrow}+F_{d_{1}}^{\uparrow \uparrow}\right)\right|_{z=0}$. The critical angle for the longitudinal energy that couples into the sediment is $62^{\circ}$, and above this angle the volume scattering is negligible. Because 


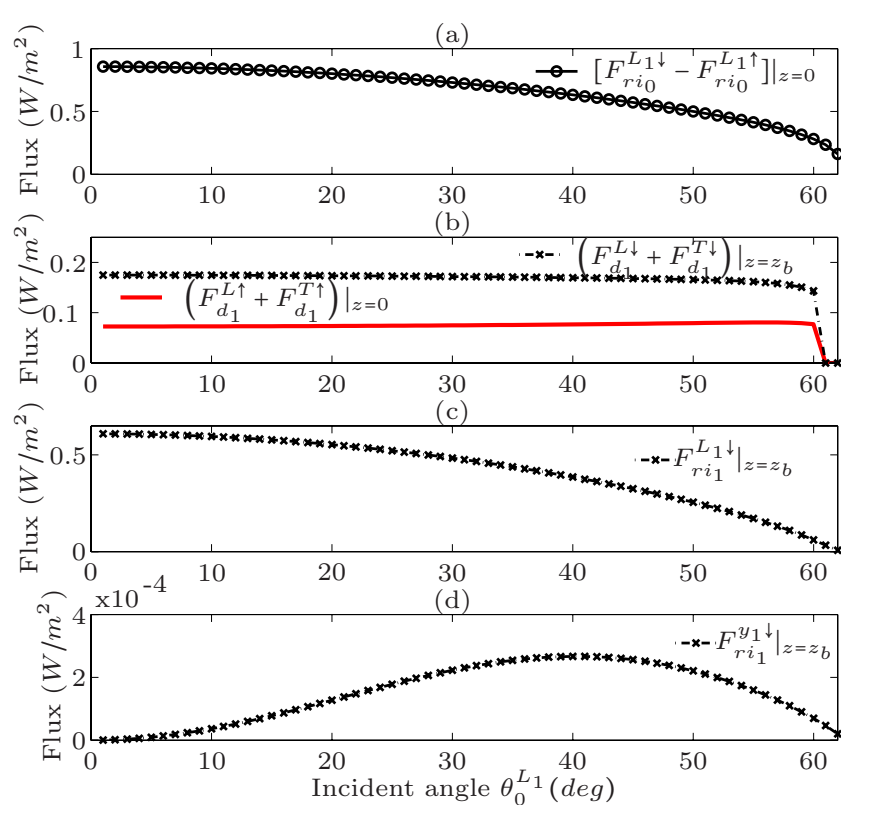

FIG. 5. (Color online) Normal component of the outgoing power flux in layer 1 for a configuration water-sand-sand and no background attenuation. (a) The difference $F_{\mathrm{ri}_{0}}^{L_{1} \downarrow}-F_{\mathrm{ri}_{0}}^{L_{1} \uparrow}$ at $z=0$ indicates the power flux that is transmitted into layer 1 from the water column. (b) Total upward and downward diffuse power fluxes at $z=0$ and $z=z_{b} \mathrm{~m}$, respectively, (c) and (d) show the power fluxes from coherent longitudinal and shear vertical energies, respectively [note different scale on (d)]. Due to the transparent boundary condition at $z=z_{b}, F_{\mathrm{ri}_{1}}^{L_{1} \uparrow}, F_{\mathrm{ri}_{1}}^{L_{2} \uparrow}, F_{\mathrm{ri}_{1}}^{L_{2} \downarrow}, F_{\mathrm{ri}_{1}}^{y_{1} \uparrow}, F_{\mathrm{ri}_{1}}^{y_{2} \uparrow}$, and $F_{\mathrm{ri}_{1}}^{y_{2} \downarrow}$ are zero.

the shear sound speed in the sediment is smaller than the sound speed in the water, there is always some minimum amount of transversal energy penetrating into the finite layer. Nevertheless, the total contribution to volume scattering related to the shear polarization is small [note scale in Fig. $5(d)]$ since the transmission coefficient from the water column into the sediment, $T_{01}^{L y}$, is very small compared to $T_{01}^{L L}$.

(a)
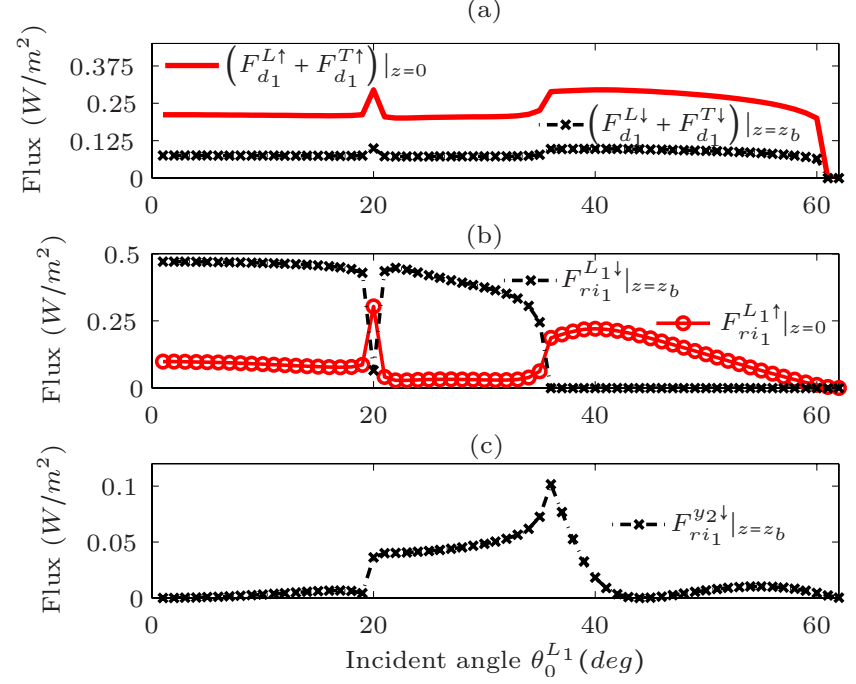

FIG. 6. (Color online) Normal component of the outgoing power flux in layer 1 for a configuration water-sand-limestone and no background attenuation. (a) Total upward and downward diffuse power fluxes at $z=0$ and $z$ $=z_{b} \mathrm{~m}$, respectively. The diffuse power flux has more variability with respect to Fig. 5(b) due to the reflection/transmission coefficients at the sandlimestone interface, (b) and (c) show the power fluxes from coherent longitudinal and shear vertical energies, respectively $\left(F_{\mathrm{ri}_{1}}^{L_{2} \uparrow}, F_{\mathrm{ri}_{1}}^{L_{2} \downarrow}, F_{\mathrm{ri}_{1}}^{y_{1} \uparrow}, F_{\mathrm{ri}_{1}}^{y_{2} \uparrow}\right.$, and $F_{\mathrm{ri}_{1}}^{y_{1} \downarrow}$ are negligible). Note that $F_{\mathrm{ri}_{0}}^{L_{1} \downarrow}-F_{\mathrm{ri}_{0}}^{L_{1} \uparrow}$ at $z=0$ (not shown) is the same as in Fig. 5.

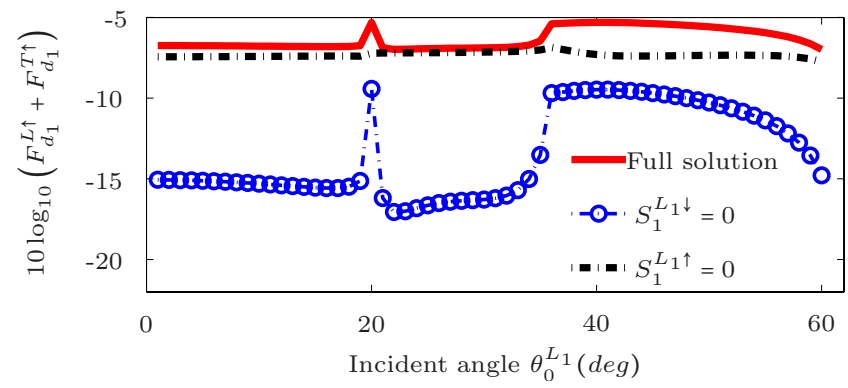

FIG. 7. (Color online) Computation of $\left.\left(F_{d_{1}}^{L \uparrow}+F_{d_{1}}^{T \uparrow}\right)\right|_{z=0}$ (upward volume scattering) with the sources $S_{1}^{L_{1} \uparrow}$ and $S_{1}^{L_{1} \downarrow}$ set to zero, as indicated. The full solution corresponds to $\left.\left(F_{d_{1}}^{L \uparrow}+F_{d_{1}}^{T \uparrow}\right)\right|_{z=0}$ from Fig. 6(a), in which all eight sources were included. See text for discussion.

In Fig. 6, the high acoustic contrast between sand and limestone results in a complex structure for $R_{12}^{L L}$ and $R_{12}^{L y}$, as shown in the reflection-transmission coefficients in Figs. 4(a) and 4(b), and the dominant coherent excitations are $I_{\mathrm{ri}_{1}}^{L_{1}}, I_{\mathrm{ri}_{1}}^{L_{1} \uparrow}$, and $I_{\mathrm{ri}_{1}}^{y_{2} \downarrow}$. Note that since $T_{01}^{L y}$ is small for the water-sediment interface, ${ }^{12}$ the coherent excitations $I_{\mathrm{ri}_{1}}^{L_{2} \uparrow}, I_{\mathrm{ri}_{1}}^{y_{1} \uparrow}, I_{\mathrm{ri}_{1}}^{L_{2} \downarrow}$, and $I_{\mathrm{ri}_{1}}^{y_{1} \downarrow}$ are negligible.

The upward source term $S_{1}^{L_{1} \uparrow}$ results in an increase in the volume scattering at $z=0$ with respect to the simulation in Fig. 5. Some of the features of these coefficients can be observed in the diffuse power flux in Fig. 6(a). For example, the sharp increase in the power flux $\left.\left(F_{d_{1}}^{L \uparrow}+F_{d_{1}}^{T \uparrow}\right)\right|_{z=0}$ at the incidence angle $\theta_{0}^{L_{1}}=20^{\circ}$ is caused by the transformation from coherent into diffuse energy of the source $I_{\mathrm{ri}_{1}}^{L_{1} \uparrow}$. The amplitude of this source is determined by the reflection coefficient $R_{12}^{L L}$, which exhibits a peak at $\theta_{1}^{L_{1}}=22.7^{\circ}$ [see Eq. (20) and Fig. 4(a)].

The contribution of each of the coherent sources can also be explored by comparing the full solution of the RT equation in Eq. (24) to the solution obtained by forcing the selected coherent source to zero. For example, Fig. 7 shows the outgoing diffuse flux $\left.\left(F_{d_{1}}^{L \uparrow}+F_{d_{1}}^{T \uparrow}\right)\right|_{z=0}$ when source terms $S_{1}^{L_{1} \uparrow}$ or $S_{1}^{L_{1} \downarrow}$ set to zero, compared to the full solution taken from Fig. 6(a). When $S_{1}^{L_{1} \downarrow}$ is zeroed, most of the power decreases, but the sharp peak at $\theta_{0}^{L_{1}}=20^{\circ}$ is still present. This indicates that most of the volume scattering is caused by the coherent intensity $I_{\mathrm{ri}_{1}}^{L_{1} \downarrow}$, while $I_{\mathrm{ri}_{1}}^{L_{1} \uparrow}$ contributes mostly at $\theta_{0}^{L_{1}}$ $=20^{\circ}$ and for $\theta_{0}^{L_{1}}>36^{\circ}$, which is when the reflection coefficient $R_{12}^{L L}$ starts to pick up at $\theta_{1}^{L_{1}}=45^{\circ}$. When $S_{1}^{L_{1} \uparrow}$ is forced to zero, most of the power remains the same except at the angles previously indicated.

A similar exploration can be done by removing the sources of coherent shear vertical intensity $S_{1}^{y_{2} \uparrow}$ or $S_{1}^{y_{2} \downarrow}$ with the result shown in Fig. 8. From this figure one can conclude that the main contribution of the shear vertical coherent intensity to the total diffuse power $\left.\left(F_{d_{1}}^{L \uparrow}+F_{d_{1}}^{T \uparrow}\right)\right|_{z=0}$ is between $\theta_{0}^{L_{1}}=21^{\circ}$ and $\theta_{0}^{L_{1}}=39^{\circ}$. Even though for this particular simulation this contribution is small, it illustrates the importance of considering shear propagation since it could be relevant for other kinds of background media such as sedimentary rock. $^{8}$

The volume scattering levels observed in Figs. 5 and 6 are unrealistic for a field measurement due to the lack of background attenuation. Figure 9 includes the attenuation 


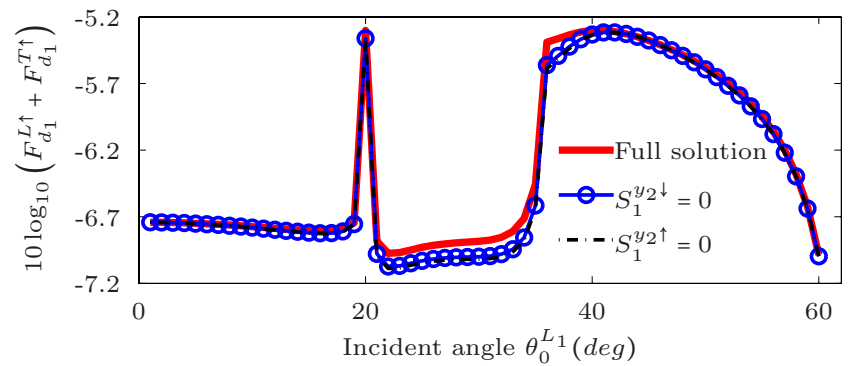

FIG. 8. (Color online) Similar to Fig. 7, with the shear vertical source terms $S_{1}^{y_{2} \uparrow}$ and $S_{1}^{y_{2} \downarrow}$ set to zero, as indicated. Again, the full solution corresponds to $\left.\left(F_{d_{1}}^{L \uparrow}+F_{d_{1}}^{T \uparrow}\right)\right|_{z=0}$ from Fig. 6(a).

from Table I for the sand layer, and this strongly reduces the amount of volume scattering as it would be expected with experimental data. In this simulation, the effect of the attenuation on the source $S_{1}^{L_{1} \uparrow}$ is stronger than for $S_{1}^{L_{1} \downarrow}$ because of a larger traveling path through the finite layer. This can be observed by comparing $F_{\text {ri1 }}^{L_{1} \uparrow}$ and $F_{\text {ril }}^{L_{1} \downarrow}$ in Fig. 9 with respect to Fig. 6. Also, the attenuation smoothes some of the sharp features in $F_{d_{1}}^{L \uparrow}+F_{d_{1}}^{T \uparrow}$ and $F_{d_{1}}^{L \downarrow}+F_{d_{1} \downarrow}^{T \downarrow}$, introduced by the reflection off the sand-limestone interface.

\section{CONCLUSION}

The transport equation for acoustics has been solved for a finite layer with random scatterers and fully elastic flat boundaries, allowing multiple conversions of longitudinal and shear coherent energy. Each interaction of the coherent energy with the boundaries of the finite layer can be used as a source for diffuse intensity, which increases the amount of volume scattering. The RT equation has been extended to include eight sources that represent all possible propagation angles for the reduced intensity when flat boundaries are assumed. The preliminary simulations presented in this paper show the flexibility of the RT formulation in isolating and interpreting the effect of geoacoustic parameters such as layering structure and background attenuation.

In this paper, the results provided by the RT theory in units of specific intensity have been converted to power flux, which is required for further comparison with experimental data. The conservation of the normal component of this flux was demonstrated with simulations of the propagation of energy within a lossless finite layer of sand.

(a)

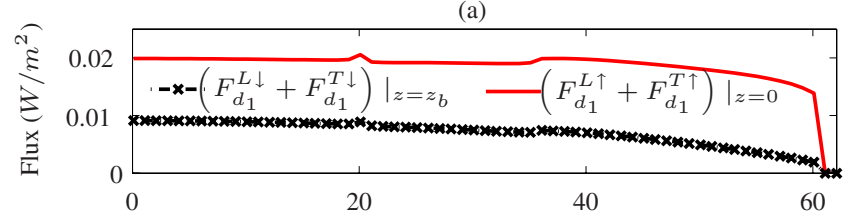

(b)

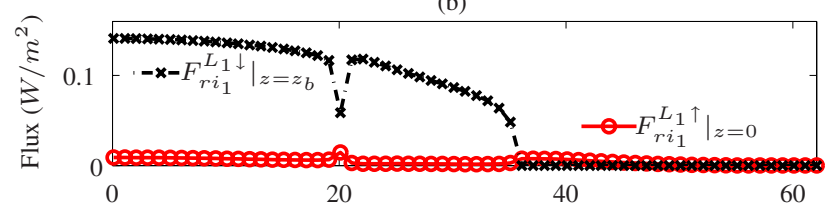

FIG. 9. (Color online) Simulation similar to Fig. 6, with attenuation $\alpha_{L}$ $=5.24$ and $\alpha_{T}=178 \mathrm{~dB} / \mathrm{m}$ in the background sand layer. Due to a longer traveling path, $F_{\mathrm{ri} 1}^{L_{1} \uparrow}$ is more strongly affected by the sediment attenuation than $F_{\mathrm{ri}}^{L_{1} \downarrow}$. The downward coherent power flux $\left.F_{\mathrm{ri}_{1}}^{y_{2} \downarrow}\right|_{z=z_{b}}$ (not shown) is negligible due to the high shear attenuation $\alpha_{T}$.
For simplicity, the RT formulation presented in this work corresponds to an environment of parallel layers of infinite extent with no lateral variation in statistical properties in the $x-y$ plane. Some of the features of the RT formulation can be appreciated under this ideal scenario, such as the incorporation of multiple discrete layers with random discrete scatterers and transformations of longitudinal and shear polarizations at the boundaries of the layers and at the scatterers. More complex scenarios of finite layers with horizontal variability have already been considered in other disciplines of electromagnetic remote sensing using the RT approach and will be used to extend the proposed model.

The simulations presented here correspond to single frequency and steady-state excitations. Nevertheless, the RT formulation can also handle arbitrary broadband excitations by means of the transient RT solution. Research on the transitory behavior of the RT is still on development in electromagnetics ${ }^{21,32}$ and acoustics ${ }^{22}$ applications. The solution of the transient RT equation is interpreted as the response of the random media to a very short impulse of energy, ${ }^{22}$ and it is argued that the solution to more complicated excitations such as linear chirps can be obtained by convolution with this "impulse" response. The extension of the presented model to transitory excitations is the subject of future research.

\section{ACKNOWLEDGMENTS}

This project has been sponsored by the Office of Naval Research (ONR), Grant No. N000140510886.

\section{APPENDIX: SOLUTION OF THE RT EQUATION BY A NUMERICAL METHOD}

Equation (24) can be solved by applying a Fourier azimuthal decomposition in $2 M+1$ terms of the Mueller matrix and the specific intensity. The corresponding Fourier series representation is

$$
\begin{aligned}
& \underline{\mathrm{P}}_{m}\left(\mu, \mu_{o}\right)=\frac{1}{2 \pi} \int_{0}^{2 \pi} \underline{\mathrm{P}}\left(\mu, \mu_{0}, \phi-\phi_{o}\right) e^{i m\left(\phi-\phi_{o}\right)} d\left(\phi-\phi_{o}\right), \\
& \underline{I}_{d m}(\mu, \tau)=\frac{1}{2 \pi} \int_{0}^{2 \pi} \underline{I}_{d}\left(\mu, \phi-\phi_{o}, \tau\right) e^{i m\left(\phi-\phi_{o}\right)} d\left(\phi-\phi_{o}\right),
\end{aligned}
$$

which allows the representation of the source terms as

$$
\underline{S}_{1 m}^{L_{1} \downarrow}(\mu)=\frac{1}{4 \pi \kappa_{T}} \underline{P}_{m}\left(\mu, \mu_{1}^{\left.L_{1}\right)}\left(\begin{array}{c}
A_{1}^{L_{1} \downarrow}\left(\mu_{1}^{L_{1}}\right) \\
0 \\
0 \\
0 \\
0
\end{array}\right)\right.
$$

for Eq. (25) and in a similar way for Eqs. (26)-(28). The $\theta$ dependency can be simplified with the Gaussian quadrature method by discretizing the variable $\theta$ in $2 N$ angles, as described by Turner and Weaver. ${ }^{11}$ Following their notation, Eq. (24) is transformed to 


$$
\begin{aligned}
\frac{\partial \mathbf{I}_{d m}(\tau)}{\partial \tau}+\mathbf{W}_{m} \mathbf{I}_{d m}(\tau) & =\mathbf{S}_{1 m}^{L_{1} \downarrow} \exp \left[-\frac{\widetilde{\sigma}_{L} \tau}{\mu_{1}^{L_{1}}}\right]+\mathbf{S}_{1 m}^{L_{2} \downarrow} \exp \left[-\frac{\widetilde{\sigma}_{L} \tau}{\mu_{1}^{L_{2}}}\right] \\
& +\mathbf{S}_{1 m}^{y_{1} \downarrow} \exp \left[-\frac{\widetilde{\sigma}_{T} \tau}{\mu_{1}^{y_{1}}}\right]+\mathbf{S}_{1 m}^{y_{2} \downarrow} \exp \left[-\frac{\widetilde{\sigma}_{T} \tau}{\mu_{1}^{y_{2}}}\right] \\
& +\mathbf{S}_{1 m}^{L_{1} \uparrow} \exp \left[\frac{\widetilde{\sigma}_{L} \tau}{\mu_{1}^{L_{1}}}\right]+\mathbf{S}_{1 m}^{L_{2}} \exp \left[\frac{\widetilde{\sigma}_{L} \tau}{\mu_{1}^{L_{2}}}\right] \\
& +\mathbf{S}_{1 m}^{y_{1} \uparrow} \exp \left[\frac{\widetilde{\sigma}_{T} \tau}{\mu_{1}^{y_{1}}}\right]+\mathbf{S}_{1 m}^{y_{2} \uparrow} \exp \left[\frac{\widetilde{\sigma}_{T} \tau}{\mu_{1}^{y_{2}}}\right],
\end{aligned}
$$

where

$$
\begin{gathered}
\mathbf{I}_{d m}(\tau)=\left[\begin{array}{c}
\underline{I}_{d m}\left(\mu_{-N}, \tau\right) \\
\underline{I}_{d m}\left(\mu_{-N+1}, \tau\right) \\
\cdot \\
\cdot \\
\cdot \\
\underline{I}_{d m}\left(\mu_{N-1}, \tau\right) \\
\underline{I}_{d m}\left(\mu_{N}, \tau\right)
\end{array}\right], \\
\mathbf{S}_{1 m}^{\left(L_{1,2} / y_{1,2}\right)(\uparrow, \downarrow)}=\left[\begin{array}{c}
\underline{\mathbf{S}}_{1 m}^{\left(L_{1,2} / y_{1,2}\right)(\uparrow, \downarrow)}\left(\mu_{-N}\right) / \mu_{-N} \\
\underline{S}_{1 m}^{\left(L_{1,2} / y_{1,2}\right)(\uparrow, \downarrow)}\left(\mu_{-N+1}\right) / \mu_{-N+1} \\
\cdot \\
\\
\cdot \\
\underline{\mathbf{S}}_{1 m}^{\left(L_{1,2} / y_{1,2}\right)(\uparrow, \downarrow)}\left(\mu_{N-1}\right) / \mu_{N-1} \\
\underline{S}_{1 m}^{\left(L_{1,2} / y_{1,2}\right)(\uparrow, \downarrow)}\left(\mu_{N}\right) / \mu_{N}
\end{array}\right],
\end{gathered}
$$

and $\mathbf{W}_{m}$ is defined by Turner and Weaver. ${ }^{11}$ The solution to Eq. (A3) consists of a particular solution for each of the eight sources and a homogeneous solution. The particular solution is

$$
\begin{aligned}
\mathbf{I}_{p m}(\tau)= & \mathbf{H}_{1 m}^{L_{1} \downarrow} \exp \left[-\frac{\widetilde{\sigma}_{L} \tau}{\mu_{1}^{L_{1}}}\right]+\mathbf{H}_{1 m}^{L_{2} \downarrow} \exp \left[-\frac{\widetilde{\sigma}_{L} \tau}{\mu_{1}^{L_{2}}}\right] \\
& +\mathbf{H}_{1 m}^{y_{1} \downarrow} \exp \left[-\frac{\widetilde{\sigma}_{T} \tau}{\mu_{1}^{y_{1}}}\right]+\mathbf{H}_{1 m}^{y_{2} \downarrow} \exp \left[-\frac{\widetilde{\sigma}_{T} \tau}{\mu_{1}^{y_{2}}}\right] \\
& +\mathbf{H}_{1 m}^{L_{1} \uparrow} \exp \left[\frac{\widetilde{\sigma}_{L} \tau}{\mu_{1}^{L_{1}}}\right]+\mathbf{H}_{1 m}^{L_{2} \uparrow} \exp \left[\frac{\widetilde{\sigma}_{L} \tau}{\mu_{1}^{L_{2}}}\right] \\
& +\mathbf{H}_{1 m}^{y_{1} \uparrow} \exp \left[\frac{\widetilde{\sigma}_{T} \tau}{\mu_{1}^{y_{1}}}\right]+\mathbf{H}_{1 m}^{y_{2} \uparrow} \exp \left[\frac{\widetilde{\sigma}_{T} \tau}{\mu_{1}^{y_{2}}}\right],
\end{aligned}
$$

where $\mathbf{D}$ is the $10 N \times 10 N$ identity matrix,

$$
\begin{aligned}
& \mathbf{H}_{1 m}^{L_{1} \downarrow}=\left(\mathbf{W}_{m}-\mathbf{D} \frac{\widetilde{\sigma}_{L}}{\mu_{1}^{L_{1}}}\right)^{-1} \mathbf{S}_{1 m}^{L_{1} \downarrow}, \\
& \mathbf{H}_{1 m}^{L_{2} \downarrow}=\left(\mathbf{W}_{m}-\mathbf{D} \frac{\widetilde{\sigma}_{L}}{\mu_{1}^{L_{2}}}\right)^{-1} \mathbf{S}_{1 m}^{L_{2} \downarrow},
\end{aligned}
$$

$$
\begin{aligned}
& \mathbf{H}_{1 m}^{y_{1} \downarrow}=\left(\mathbf{W}_{m}-\mathbf{D} \frac{\widetilde{\sigma}_{T}}{\mu_{1}^{y_{1}}}\right)^{-1} \mathbf{S}_{1 m}^{y_{1} \downarrow}, \\
& \mathbf{H}_{1 m}^{y_{2} \downarrow}=\left(\mathbf{W}_{m}-\mathbf{D} \frac{\widetilde{\sigma}_{T}}{\mu_{1}^{y_{2}}}\right)^{-1} \mathbf{S}_{1 m}^{y_{2} \downarrow}
\end{aligned}
$$

correspond to the downward source terms and

$$
\begin{aligned}
& \mathbf{H}_{1 m}^{L_{1} \uparrow}=\left(\mathbf{W}_{m}+\mathbf{D} \frac{\widetilde{\sigma}_{L}}{\mu_{1}^{L_{1}}}\right)^{-1} \mathbf{S}_{1 m}^{L_{1} \uparrow}, \\
& \mathbf{H}_{1 m}^{L_{2} \uparrow}=\left(\mathbf{W}_{m}+\mathbf{D} \frac{\widetilde{\sigma}_{L}}{\mu_{1}^{L_{2}}}\right)^{-1} \mathbf{S}_{1 m}^{L_{2} \uparrow},
\end{aligned}
$$

$$
\begin{aligned}
& \mathbf{H}_{1 m}^{y_{1} \uparrow}=\left(\mathbf{W}_{m}+\mathbf{D} \frac{\widetilde{\sigma}_{T}}{\mu_{1}^{y_{1}}}\right)^{-1} \mathbf{S}_{1 m}^{y_{1} \uparrow}, \\
& \mathbf{H}_{1 m}^{y_{2} \uparrow}=\left(\mathbf{W}_{m}+\mathbf{D} \frac{\widetilde{\sigma}_{T}}{\mu_{1}^{y_{2}}}\right)^{-1} \mathbf{S}_{1 m}^{y_{2} \uparrow}
\end{aligned}
$$

correspond to the upward terms.

The homogeneous solution can be found by solving an eigenvalue problem with eigenvectors $g_{m n}$ and eigenvalues $\lambda_{m n}$. The full solution for the $m$ th Fourier expansion term of the diffuse intensity is written as

$$
\mathbf{I}_{d m}(\tau)=\mathbf{I}_{p m}(\tau)+\sum_{n=1}^{10 N} C_{m n} g_{m n} e^{-\lambda_{m n} \tau}
$$

where the constants $C_{m n}$ must be found from the boundary conditions.

The intensity vector $\mathbf{I}_{d m}$ can be divided into upward $\left(\mathbf{I}_{d m}^{+}, \mu<0\right)$ and downward $\left(\mathbf{I}_{d m}^{-}, \mu>0\right)$ intensities, and it is evaluated at $\tau=0^{+}$and $\tau=\tau_{b}^{-}$, so the top boundary condition $\mathbf{I}_{d m}^{-}\left(\tau=0^{+}\right)=\hat{\mathbf{R}}_{10} \mathbf{I}_{d m}^{+}\left(\tau=0^{+}\right)$yields the equation

$$
\sum_{n=1}^{10 N} C_{m n} g_{m n}^{-}+\mathbf{I}_{p m}^{-}\left(0^{+}\right)=\hat{\mathbf{R}}_{10}\left(\sum_{n=1}^{10 N} C_{m n} g_{m n}^{+}+\mathbf{I}_{p m}^{+}\left(0^{+}\right)\right),
$$

and the bottom boundary condition $\mathbf{I}_{m}^{+}\left(\tau_{b}^{-}\right)=\hat{\mathbf{R}}_{12} \mathbf{I}_{m}^{-}\left(\tau_{b}^{-}\right)$yields

$$
\begin{aligned}
& \sum_{n=1}^{10 N} C_{m n} g_{m n}^{+} e^{-\lambda_{m n} \tau_{b}}+\mathbf{I}_{p m}^{+}\left(\tau_{b}\right) \\
& =\hat{\mathbf{R}}_{12}\left(\sum_{n=1}^{10 N} C_{m n} g_{m n}^{-} e^{-\lambda_{m n} \tau_{b}}+\mathbf{I}_{p m}^{-}\left(\tau_{b}\right)\right),
\end{aligned}
$$

where

$$
\hat{\mathbf{R}}_{10}=\left(\begin{array}{ccccccc}
0 & 0 & 0 & \cdots & 0 & 0 & \underline{\hat{\mathbf{R}}}_{10}\left(\mu_{-1}\right) \\
0 & 0 & 0 & \cdots & 0 & \underline{\underline{\mathbf{R}}}_{10}\left(\mu_{-2}\right) & 0 \\
& & & & . & & \\
& & & . & & & \\
0 & \underline{\hat{\mathbf{R}}}_{10}\left(\mu_{-N+1}\right) & 0 & 0 & 0 & 0 & 0 \\
\underline{\hat{\mathbf{R}}}_{10}\left(\mu_{-N}\right) & 0 & 0 & 0 & 0 & 0 & 0
\end{array}\right) \text {, }
$$


$\hat{\mathbf{R}}_{12}=\left(\begin{array}{ccccccc}0 & 0 & 0 & \cdots & 0 & 0 & \underline{\hat{\mathbf{R}}}_{12}\left(\mu_{N}\right) \\ 0 & 0 & 0 & \cdots & 0 & \underline{\hat{\mathbf{R}}}_{12}\left(\mu_{N-1}\right) & 0 \\ & & & & \cdot & & \\ & & & \cdot & & & \\ 0 & \underline{\hat{\mathbf{R}}}_{12}\left(\mu_{2}\right) & 0 & 0 & 0 & 0 & 0 \\ \hat{\underline{\hat{R}}}_{12}\left(\mu_{1}\right) & 0 & 0 & 0 & 0 & 0 & 0\end{array}\right)$,

(A13)

with the matrix of reflection coefficients $\hat{\mathbf{R}}_{a b}$ defined as

$$
\hat{\mathbf{R}}_{a b}=\left(\begin{array}{ccccc}
\hat{R}_{a b}^{L L} & \hat{R}_{a b}^{y L} & 0 & 0 & 0 \\
\hat{R}_{a b}^{L y} & \hat{R}_{a b}^{y y} & 0 & 0 & 0 \\
0 & 0 & \hat{R}_{a b}^{x x} & 0 & 0 \\
0 & 0 & 0 & \hat{R}_{a b}^{U U} & \hat{R}_{a b}^{V U} \\
0 & 0 & 0 & \hat{R}_{a b}^{U V} & \hat{R}_{a b}^{V V}
\end{array}\right) .
$$

Equations (A10) and (A11) can be written in matrix form as

$$
\begin{aligned}
& G^{-}(0) C_{m}+\mathbf{I}_{p m}^{-}(0)=\widehat{\mathbf{R}_{10}}\left(G^{+}(0) C_{m}+\mathbf{I}_{p m}^{+}(0)\right), \\
& G^{+}\left(\tau_{b}\right) C_{m}+\mathbf{I}_{p m}^{+}\left(\tau_{b}\right)=\widehat{\mathbf{R}_{12}}\left(G^{-}\left(\tau_{b}\right) C_{m}+\mathbf{I}_{p m}^{-}\left(\tau_{b}\right)\right),
\end{aligned}
$$

where

$$
\begin{aligned}
& G^{-}(\tau)=\left(\begin{array}{cccc}
g_{m 1}^{1} e^{-\lambda_{1} \tau} & g_{m 2}^{1} e^{-\lambda_{2} \tau} & \ldots & g_{m_{10} N}^{1} e^{-\lambda_{10 N} \tau} \\
g_{m 1}^{2} e^{-\lambda_{1} \tau} & g_{m 2}^{2} e^{-\lambda_{2} \tau} & \ldots & g_{m 10 N}^{2} e^{-\lambda_{10 N} \tau} \\
\cdot & \cdot & \cdot & \cdot \\
g_{m 1}^{N} e^{-\lambda_{1} \tau} & g_{m 2}^{N} e^{-\lambda_{2} \tau} & \ldots & g_{m N}^{N} e^{-\lambda_{10 N} \tau}
\end{array}\right), \\
& G^{+}(\tau)=\left(\begin{array}{cccc}
g_{m 1}^{-N} e^{-\lambda_{1} \tau} & g_{m 2}^{-N} e^{-\lambda_{2} \tau} & \ldots & g_{m 10^{N}}^{-N} e^{-\lambda_{10 N} \tau} \\
g_{m 1}^{-N+1} e^{-\lambda_{1} \tau} & g_{m 2}^{-N+1} e^{-\lambda_{2} \tau} & \ldots & g_{m 10 N}^{-N+1} e^{-\lambda_{10 N} \tau} \\
\cdot & \cdot & \cdot & \cdot \\
g_{m 1}^{-1} e^{-\lambda_{1} \tau} & g_{m 2}^{-1} e^{-\lambda_{2} \tau} & \ldots & g_{m N}^{-1} e^{-\lambda_{10 N} \tau}
\end{array}\right), \\
& C_{m}=\left(\begin{array}{c}
\mathrm{A} 16) \\
C_{m 1} \\
C_{m 2} \\
\ldots \\
C_{m 10 N}
\end{array}\right) .
\end{aligned}
$$

The equations in Eq. (A15) are grouped to form a single matrix equation

$$
G_{1} C_{m}+P=R_{G} C_{m}+R_{P},
$$

where

$$
\begin{aligned}
& G_{1}=\left(\begin{array}{c}
G^{-}(0) \\
G^{+}\left(\tau_{b}\right)
\end{array}\right), \\
& P=\left(\begin{array}{c}
\mathbf{I}_{p m}^{-}(0) \\
\mathbf{I}_{p m}^{+}\left(\tau_{b}\right)
\end{array}\right), \\
& R_{G}=\left(\begin{array}{c}
\hat{\mathbf{R}}_{10} G^{+}(0) \\
\hat{\mathbf{R}}_{12} G^{-}\left(\tau_{b}\right)
\end{array}\right),
\end{aligned}
$$

$$
R_{P}=\left(\begin{array}{c}
\hat{\mathbf{R}}_{10} \mathbf{I}_{p m}^{+}(0) \\
\hat{\mathbf{R}}_{12} \mathbf{I}_{p m}^{-}\left(\tau_{b}\right)
\end{array}\right) .
$$

Solving for $C_{m}$,

$$
C_{m}=\left(G_{1}-R_{G}\right)^{-1}\left(R_{P}-P\right) .
$$

${ }^{1}$ D. R. Jackson and M. D. Richardson, High-Frequency Seafloor Acoustics, 1st ed. (Springer, New York, 2007).

${ }^{2}$ D. R. Jackson, D. P. Winebrenner, and A. Ishimaru, "Application of the composite roughness model to high-frequency bottom scattering," J. Acoust. Soc. Am. 79, 1410-1422 (1986).

${ }^{3} \mathrm{~A}$. N. Ivakin, "A unified approach to volume and roughness scattering," J. Acoust. Soc. Am. 103, 827-837 (1998).

${ }^{4}$ D. Tang, "Acoustic wave scattering from a random ocean bottom," Ph.D. thesis, Massachusetts Institute of Technology and Woods Hole Oceanographic Institution, Massachusetts (1991).

${ }^{5}$ P. D. Mourad and D. R. Jackson, "A model/data comparison for lowfrequency bottom backscatter," J. Acoust. Soc. Am. 94, 344-358 (1993).

${ }^{6}$ A. P. Lyons, "The potential impact of shell fragment distributions on highfrequency seafloor backscatter," J. Oceanic Eng. 30, 843-851 (2005).

${ }^{7}$ D. Chu, K. L. Williams, D. Tang, and D. R. Jackson, "High-frequency bistatic scattering by sub-bottom gas bubbles," J. Acoust. Soc. Am. 102, 806-814 (1997).

${ }^{8}$ A. N. Ivakin and D. R. Jackson, "Effects of shear elasticity on sea bed scattering: Numerical examples," J. Acoust. Soc. Am. 103, 346-354 (1998).

${ }^{9}$ A. A. Kokhanovsky, Optics of Light Scattering Media, 2nd ed. (SpringerPraxis, U.K., 2001).

${ }^{10}$ L. Margerin, M. Campillo, and B. van Tiggelen, "Radiative transfer and diffusion of waves in a layered medium: New insight into coda Q," Geophys. J. Int. 134, 596-612 (1998).

${ }^{11}$ J. A. Turner and R. L. Weaver, "Radiative transfer of ultrasound," J. Acoust. Soc. Am. 96, 3654-3672 (1994).

${ }^{12}$ J. E. Quijano and L. M. Zurk, "Application of radiative transfer theory to acoustic propagation in the ocean bottom," in Proceedings of the Oceans '07, Vancouver, BC, Canada (2007), pp. 1-7.

${ }^{13}$ A. Ishimaru, Wave Propagation and Scattering in Random Media, 1st ed. (Academic, New York, 1978), Vol. 1.

${ }^{14}$ M. I. Mishchenko and L. D. Travis, Multiple Scattering of Light by Particles, 1st ed. (Cambridge University Press, Cambridge, 2006).

${ }^{15} \mathrm{~L}$. Tsang and A. Ishimaru, "Radiative wave and cyclical transfer equations for dense non tenuous media," J. Opt. Soc. Am. 2, 2187-2194 (1985).

${ }^{16}$ B. Wen, L. Tsang, D. P. Winebrenner, and A. Ishimaru, "Dense medium radiative transfer theory: Comparison with experiment and application to microwave remote sensing and polarimetry," IEEE Trans. Geosci. Remote Sens. 28, 46-59 (1990).

${ }^{17}$ L. M. Zurk, L. Tsang, and D. P. Winebrenner, "Scattering properties of dense media from Monte Carlo simulations with application to active remote sensing of snow," Radio Sci. 31, 803-819 (1996).

${ }^{18} \mathrm{G}$. Bal, "Radiative transfer equations with varying refractive index: A mathematical perspective," J. Opt. Soc. Am. 23, 1639-1644 (2006).

${ }^{19} \mathrm{~K}$. N. Liou, Introduction to Atmospheric Radiation, 2nd ed. (Academic, San Diego, CA, 2002).

${ }^{20} \mathrm{G}$. A. Titov, "Radiative horizontal transport and absorption in stratocumulus clouds," J. Atmos. Sci. 55, 2549-2560 (1998).

${ }^{21}$ T. Okutucu, Y. Yener, and A. A. Busnaina, "Transient radiative transfer in participating media with pulse-laser irradiation: An approximate Galerkin solution," J. Quant. Spectrosc. Radiat. Transf. 103, 118-130 (2007).

${ }^{22}$ J. A. Turner and R. L. Weaver, "Time dependence of multiply scattered diffuse ultrasound in polycrystalline media," J. Acoust. Soc. Am. 97, 2639-2644 (1995).

${ }^{23}$ S. Chandrasekhar, Radiative Transfer, 1st ed. (Dover, New York, 1960).

${ }^{24}$ R. T. Shin and J. A. Kong, "Radiative transfer theory for active remote sensing of a homogeneous layer containing spherical scatterers," J. Appl. Phys. 52, 4221-4230 (1981).

${ }^{25}$ L. Tsang, J. A. Kong, and R. T. Shin, Theory of Microwave Remote Sensing, 1st ed. (Wiley-Interscience, New York, 1985).

${ }^{26}$ L. M. Brekhovskikh, Waves in Layered Media, 2nd ed. (Academic, Orlando, FL, 1980). 
${ }^{27}$ J. A. Turner and R. L. Weaver, "Ultrasonic radiative transfer in polycrystalline media: Effects of a fluid-solid interface," J. Acoust. Soc. Am. 98, 2801-2808 (1995).

${ }^{28}$ D. R. Jackson, K. B. Briggs, K. L. Williams, and M. D. Richardson, "Tests of models for high-frequency seafloor," J. Oceanic Eng. 21, 458-470 (1996).

${ }^{29}$ M. J. Buckingham, "Wave propagation, stress relaxation, and grain-tograin shearing in saturated, unconsolidated marine sediments," J. Acoust.
Soc. Am. 108, 2796-2815 (2000).

${ }^{30}$ E. L. Hamilton, "Geoacoustic modeling of the sea floor," J. Acoust. Soc. Am. 68, 1313-1339 (1980).

${ }^{31}$ E. L. Hamilton, " $V_{p} / V_{s}$ and Poisson's ratios in marine sediments and rocks," J. Acoust. Soc. Am. 66, 1093-1101 (1979).

${ }^{32} \mathrm{C}$. Wu, "Propagation of scattered radiation in a participating planar medium with pulse irradiation,” J. Quant. Spectrosc. Radiat. Transf. 64, 537548 (2000). 